RESEARCH ARTICLE

\title{
A Wire-Driven Discrete Continuum Robot Sliding and Non-sliding Backbone Design Analysis and Validation of Kinematics/Kinetics
}

\author{
Azamat Yeshmukhametov, ${ }^{1,2}$ Zholdas Buribayev, ${ }^{2}$ Beibut Amirgaliyev, ${ }^{3}$ and Yedilkhan Amirgaliyev,
}

${ }^{1}$ Department of Robotics and Engineering tools of Automation, Satbayev University, Almaty 050000, Kazakhstan.

${ }^{2}$ Department of computer science, Kazakh National University, Almaty, 050000, Kazakhstan

${ }^{3}$ Astana IT University, Nur-Sultan city, 010000, Kazakhstan

${ }^{4}$ Academy Science of Kazakhstan, Institute of Information and Computational Technologies , Kazakhstan, Almaty, 050000, Kazakhstan

Correspondence should be addressed to Azamat Yeshmukhametov; yeshmukhametov.coba@gmail.com

\section{(C) Higher Education Press 2020}

\begin{abstract}
Wire-driven hyper-redundant continuum manipulators are gaining more popularity and finding more applications in industry and in minimally invasive surgery. Unlike traditional rigid link manipulators, continuum robots with a flexible backbone structure are able to work in a highly constrained workspace and in an unstructured environment. However, in spite of a possible wide range of reachability, continuum manipulators have some issues related to payload capacity, accuracy and control. Therefore, in this research, we propose a novel hyper-redundant continuum robot with a passive sliding disc mechanism to improve payload capacity and accuracy. To prove the sliding mechanism concept, we demonstrate a comparison analysis with a conventional non-sliding continuum robot arm in a payload test, a bending test and a reachability test. Moreover, with this novel design, we are proposing robot kinematics and kinetic formulation and simulation results to validate the effectiveness of the sliding disc mechanism.
\end{abstract}

Keywords sliding disc, continuum robot, robot backbone, wire-driven, kinematics and kinetics, wiretension, design analysis.

\section{Introduction}

Hyper-redundant wire-driven continuum manipulators are very attractive to the scientific research community. They can be utilized in applications for the nuclear industry, chemical plants or the medical field, all of which demand work in a confined workspace and an extremely constrained environment. Their unique capability could help us reach new horizons of robot application.

Despite their advantages, continuum manipulators have lower accuracy than traditional rigid type robots because of their high structural redundancy [1]. To improve continuum robot precision, previous research has attempted to formulate its kinematics by assuming a piecewise constant curvature [2,3]. However, the curvature in actual robotic systems is not constant and depends on position and posture.

Actuation of continuum manipulators could either be indirect on power transmission (as pneumatic and hydraulic [4,5]) or direct through mechanical transmission (via tendon/wire/cable [6-9]). Among the actuation systems classified, wire-driven continuum manipulators will be potentially applicable in areas such as industry, medicine and agriculture. In terms of payload capacity and continuum manipulators, it also depends on the actuation system. Wire-driven actuation is often used in applications in which substantial accuracy and payload capacity are required. There are two types of wire-driven continuum manipulators: hard continuum manipulators [10-12] and discrete hyperredundant continuum manipulators [13-15]. The backbones of the former type are mostly made of elastic material (e.g. NiTi) and such a design allows it to bend at any point of the structure. The latter type of continuum manipulators can bend only at the joints because the spacer discs are interconnected by universal or ball joints. In this research, we deal with wire-driven discrete continuum robots 
classified as the latter type with a passive sliding backbone disc mechanism. Similar research with sliding spacer discs was conducted by Burgner-Kahrs et.al. They developed a tendon-driven continuum robot with extendable sections using permanent magnets. The robot sections extend or contract by using an additional motor and another three motors to guide the manipulator bending. For extension, magnets move the discs by their repulsion force, while maintaining a certain distance from each other [16]. However, in our proposed design, the manipulator does not extend or contract. Instead, the sliding mechanism is designed to make internal strain stress evenly distributed along the backbone structure.

Furthermore, Kang and co-workers also presented a similar robot interlaced continuum robot arm with a sliding spacer disc along the backbone in the principle of following a master. However, the proposed design has a complicated structure mostly suited for inspection tasks only [17].

Continuum manipulators have a problem related to twist deformation. During the motion, the external load or impact might cause twist deformation. So, to avoid problems caused by twist deformation, a wire-driven robot arm with constrained spherical joints has been proposed for minimally invasive surgery[18]. Kai Xu also proposed a surgical robot with redundant backbones and constrained bending curvature for continuously variable stiffness. Additionally, Xu developed a unique actuation system for continuum manipulators, which prevents them from buckling under pressure [19].

There are many kinematic models for continuum manipulators based on the robot structure. The most popular kinematic model is the constant curvature model, which simplifies formulation by assuming the robot backbone kinematics in a planar deformed state. The great contribution on continuum robots kinematics was made by Hannan and Walker, who proposed kinematics applicable for concentric manipulators and hyper-redundant as well. Furthermore, to improve continuum robot stiffness, Walker and Hannan proposed kinematics of continuum manipulator designed with springs [22], for the manipulators with pneumatic actuation Ohno and Hirose method [23], and for the flexible backboned manipulators Gravagne's method [24]. The derived kinematic model for the wire-driven continuum robot arm was also proposed by Zheng Li et al. [25].

In this research, we conducted the motion simulations that combine kinematics with robot kinetics because the proposed continuum manipulator design with sliding spacer disc mechanism requires torque/force equilibrium equations to determine the positions of sliding discs that passively slide along the backbone. It also formulates the way to control the tip-position.

The main contribution of this paper is the proposal of a new wire-driven continuum robot with the following beneficial features:

- Passive bending stress distribution. During the bending motion, discs passively slide along the backbone in order to avoid a bending torque concentrating on a certain joint.

- Simultaneous wire tension control with a proposed pre-tension mechanism.

- Improved payload capacity.

The remainder of this paper is organized as follows: design concept, design analysis, and proof of the sliding backbone disc concept.

\section{Robot design}

\subsection{Continuum manipulator design}

The design of the proposed manipulator is demonstrated in Fig.1. In this research, we modified a previous prototype with the non-sliding backbone disc (Fig. 2b). TakoBot with sliding discs consists of three parts: the continuum part, the pre-tension part, and the control box. The continuum part, the slender part of the robot, consists of two independent sections. Each section has five segments separated by discs that slide along a backbone shaft. The neighboring two rods are interconnected by a universal joint (Fig.2). Four compression springs are allocated at every 90 degrees between adjacent discs to transfer forces and are firmly fixed on the disc surface by circular pins. The discs, except for one that separates the first and second section, slide along the backbone through the linear bearing equipped in the disc center. 


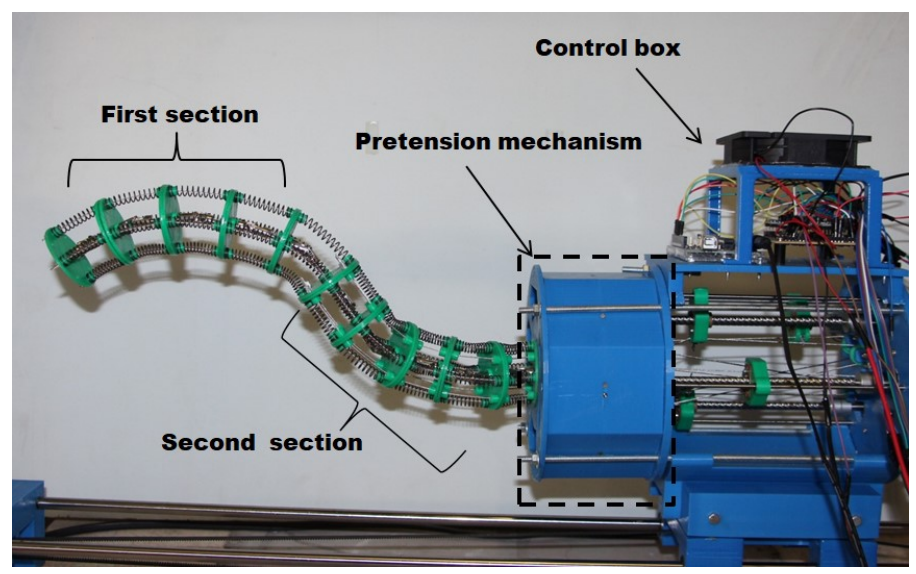

Fig. 1 Overall appearance of TakoBot.

Fig. 2 shows the sliding backbone disc design. The mechanism endows two degrees of freedom into every disc. One is a translational motion along the backbone axis, and the other is a rotational motion around the same axis.

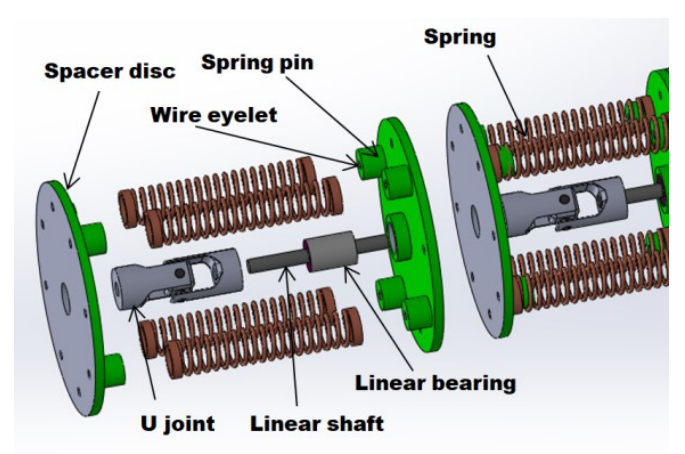

(a) Sliding

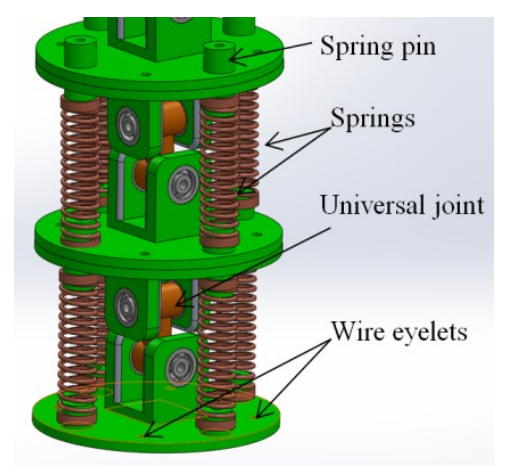

(b) Non-sliding model

Fig.2 Single segment of sliding structure (a) and non-sliding model (b)

The previous non-sliding prototype was inspired by the Elephant Trunk robot (Walker and Hanna, 1999) where Walker used cable-routing spacer discs, universal joints and extension springs [20].

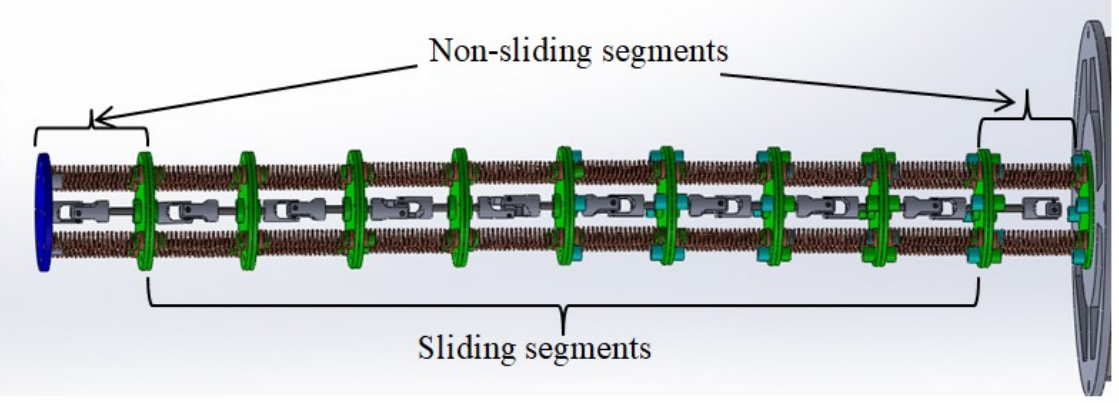

Fig.3 Slender part architecture of TakoBot 


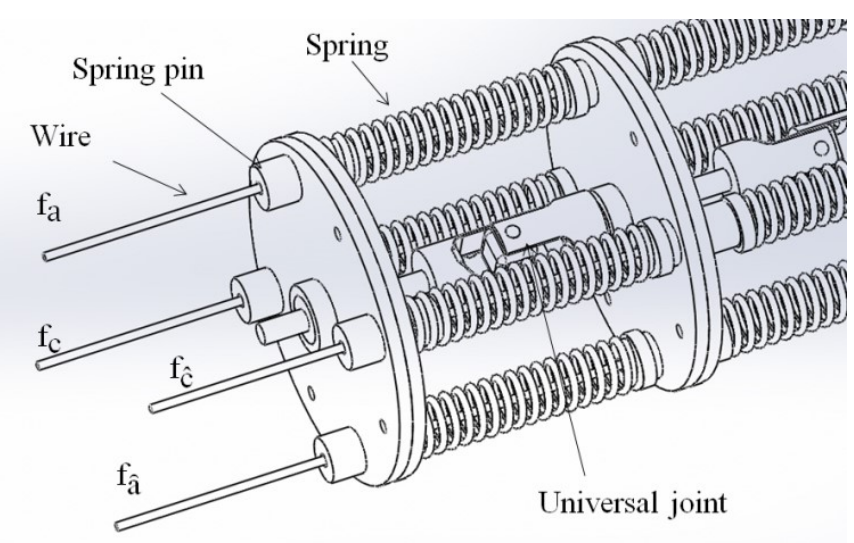

Fig. 4 Wire routing arrangement

As it shown in Fig. 3, the first and the last discs do not slide, which means that the total length of the continuum part always remains constant. As shown in Fig.4, the wires pass through the inside of the springs. This wire and spring arrangement makes the wire tension vector and spring force vector almost parallel, which will avoid yielding to unnecessary torque that might occur if the two vectors are not parallel.

\subsection{Pretension mechanism design}

Pretension mechanism (PtM) is a passive mechanical device that compensates for wire tension in any possible posture the robot takes. The PtM device is required for the actuation system that TakoBot uses. This system uses a pair of wires driven by one motor and multiple pairs of wires are used to control it. The PtM device might be unnecessary if one motor controls one cable individually, but such a design inevitably conduces a complexity in control.

All motors must be driven almost all the time during operation in order to keep a certain level of tension in every wire by monitoring tension with sensors and relaying that information back to the controller.

Also, the PtM contributes to stabilizing the motion of the manipulator by holding a certain level of tension of all wires which avoids the sag effect.

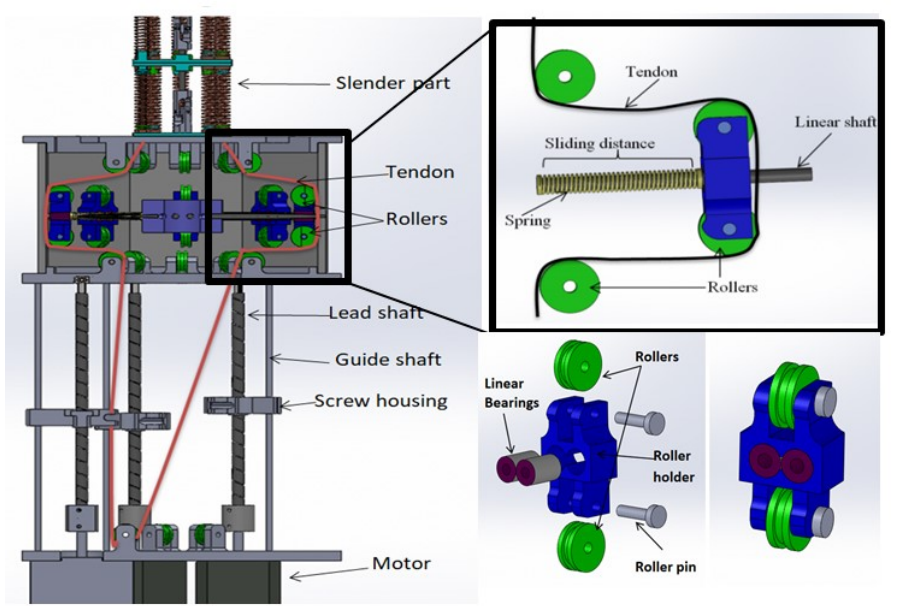

Fig. 5 Wire routing schematics inside the pretension mechanism

A tendon passes through the PtM device as shown in Fig. 5. The PtM device is equipped with rollers to prevent friction between the cable and robot parts. The PtM consists of several components: PtM holder, inner base, springs, linear guide shaft and roller holders. The inner base is connected to the PtM holder by the linear guide shaft and the roller holder slides along the linear shaft. Wire tension is generated by compression springs along the linear shaft (Fig. 6,7). If high tension occurs on a wire, the roller holder gets close to the inner base. Thus, the PtM device can compensate tension for eight cables simultaneously with no electronic devises being required for operation, which will enhance device reliability. Detailed analysis of pre-tension mechanism is here [26]. In order to avoid a spring buckling 
effect, the spring constant of pretension mechanism spring and single segment spring constant value should be equal.

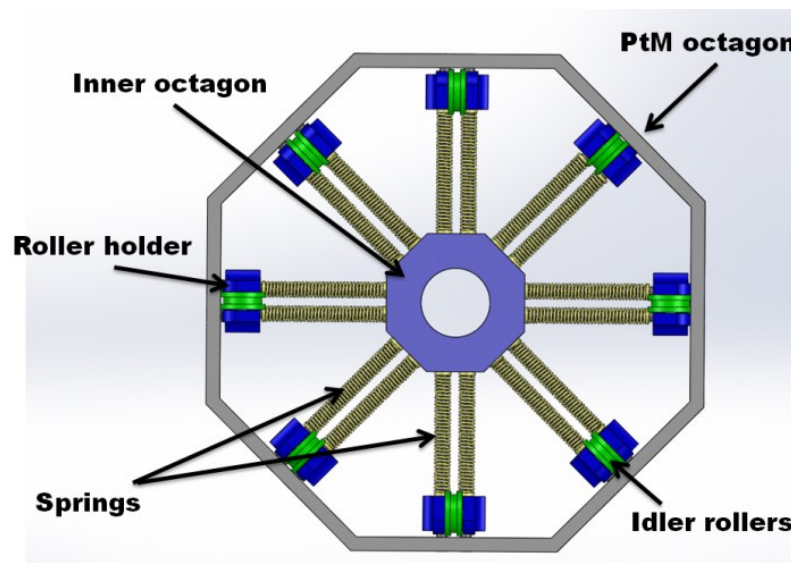

Fig.6 Pretension mechanism CAD model

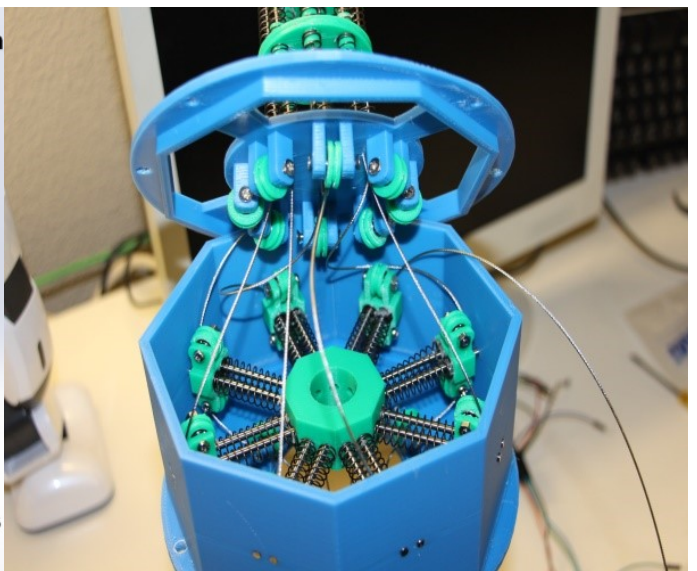

Fig.7 Fabricated prototype

\subsection{Actuation system}

TakoBot employs long lead screw ( $\varphi 5 \mathrm{~mm}$, lead $6 \mathrm{~mm}$ ) that transfer the rotation of the individual stepping motor to linear operation of wire. Pair of wires is actuated by one motor using the push-pull principle. Four motors are used in total: two for the first section and two other motors for the second section. We use Hybrid Bipolar Stepping Motors with $0.49 \mathrm{~N} / \mathrm{m}$ torque (Trinamic Motion Control, Inc.). The total shaft stroke is $150 \mathrm{~mm}$. The linear guide shaft prevents the screw housing from twisting/ rotating around the screw rod (Fig. 8).

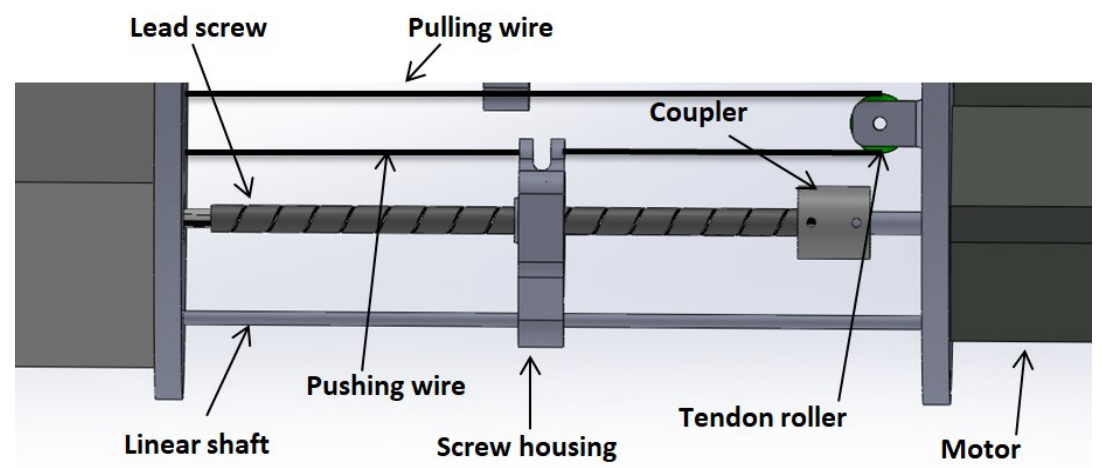

Fig.8 TakoBot wire actuating system

\subsection{Design comparison analysis}

The backbone is a crucial part of continuum manipulators. Therefore, based on the backbone structure, robot characteristics and capabilities may be different. One of the main reasons for using continuum manipulators is potential payload capacity and bending capability. For identification of the best robot backbone design, we manufactured two prototypes (with sliding discs and without sliding discs) (Table 1) and conducted several tests to figure out the optimal continuum robot design. In all prototypes, we used the same springs and motors.

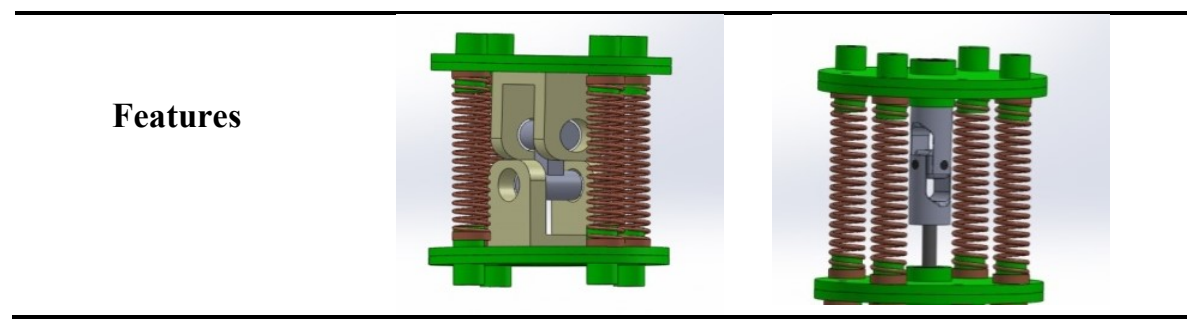




\begin{tabular}{ccc}
\hline Name & Non-sliding & Sliding \\
Segment size & Length: $35 \mathrm{~mm}$ \\
Diameter: $50 \mathrm{~mm}$ & $\begin{array}{c}\text { Length } 35 \mathrm{~mm} \\
\text { Diameter: } 50 \mathrm{~mm}\end{array}$ \\
$\begin{array}{c}\text { Num. of segments } \\
\text { Max. bending angle }\end{array}$ & 10 & 10 \\
Torsional motion & $<45^{0}$ & $<45^{0}-50^{0}$ \\
$\begin{array}{c}\text { Required Torque } \\
\text { Max. payload } \\
\text { capacity }\end{array}$ & $\mathrm{no}$ & yes \\
Control & $175 \mathrm{~g}$ & $\tau<0.69 \mathrm{~N} \cdot \mathrm{m}$ \\
\hline & Difficult & $220 \mathrm{~g}$ \\
\hline
\end{tabular}

Table 1. TakoBot prototypes comparison table

\subsubsection{Test on payload capacity}
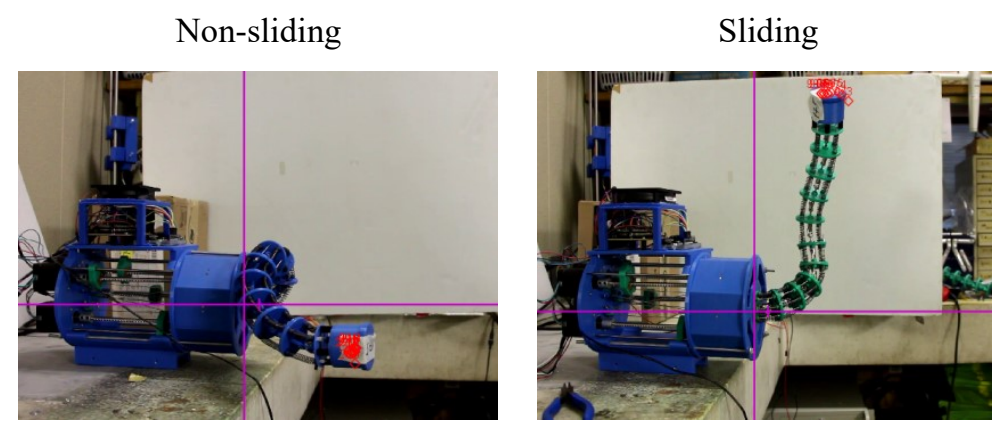

Fig.9 Payload test experimental final robot outputs

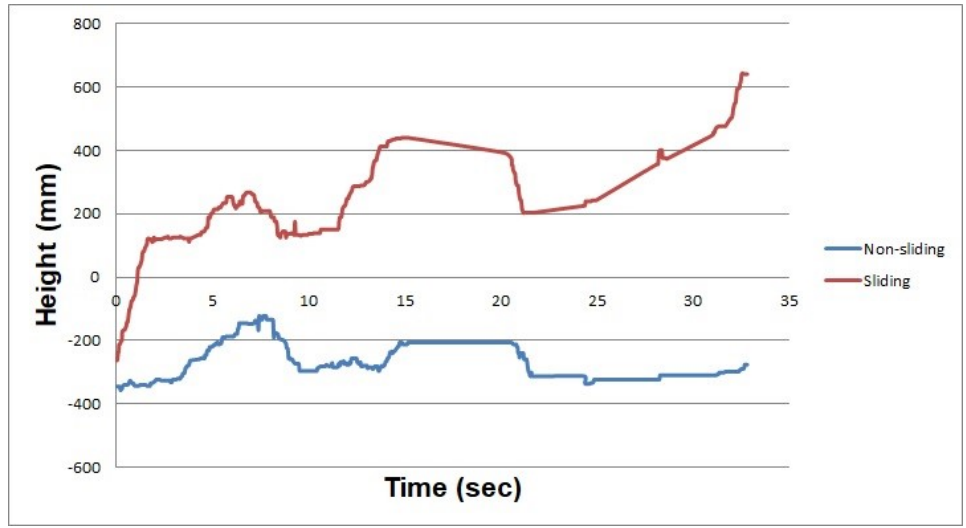

Fig.10 Weight manipulability comparison graph

To conduct payload tests of both prototypes, we gave the same task with the same motor. In this experimental setup, we put $175 \mathrm{~g}$ weight at the tip (Fig 9).The height trajectory of the tip is shown in Fig. 10. In this experiment, we used a tracker to measure the manipulation trajectory and height, and designed a special code to lift the load as height as possible. For the both prototype same code had been used and motor torque was same as well. The better payload capacity feature was observed in the case of sliding discs, which is explained by the following statements:

1. The sliding backbone can distribute applied force/torque equally to all of the segments.

2. In the non-sliding prototype, much of force/torque concentrated on the bending segment that the wires directly connect, and the sharply bent segment consumes more power than the sliding backbone (Fig 11).

\subsubsection{Test on basic motions}

First, we tested the simple bending operation to observe the difference of the resultant shape of manipulator between the TakoBot and the prototype with non-sliding discs under the identical motor driving (Fig.11). Next, we tested for " $\mathrm{S}$ " shaped motion as shown in Fig.12. For continuum manipulators, must be able to form an S-shape when in operation. As shown in Fig.12 TakoBot demonstrated more prominent ' $\mathrm{S}$ ' bending with more small curvature. 

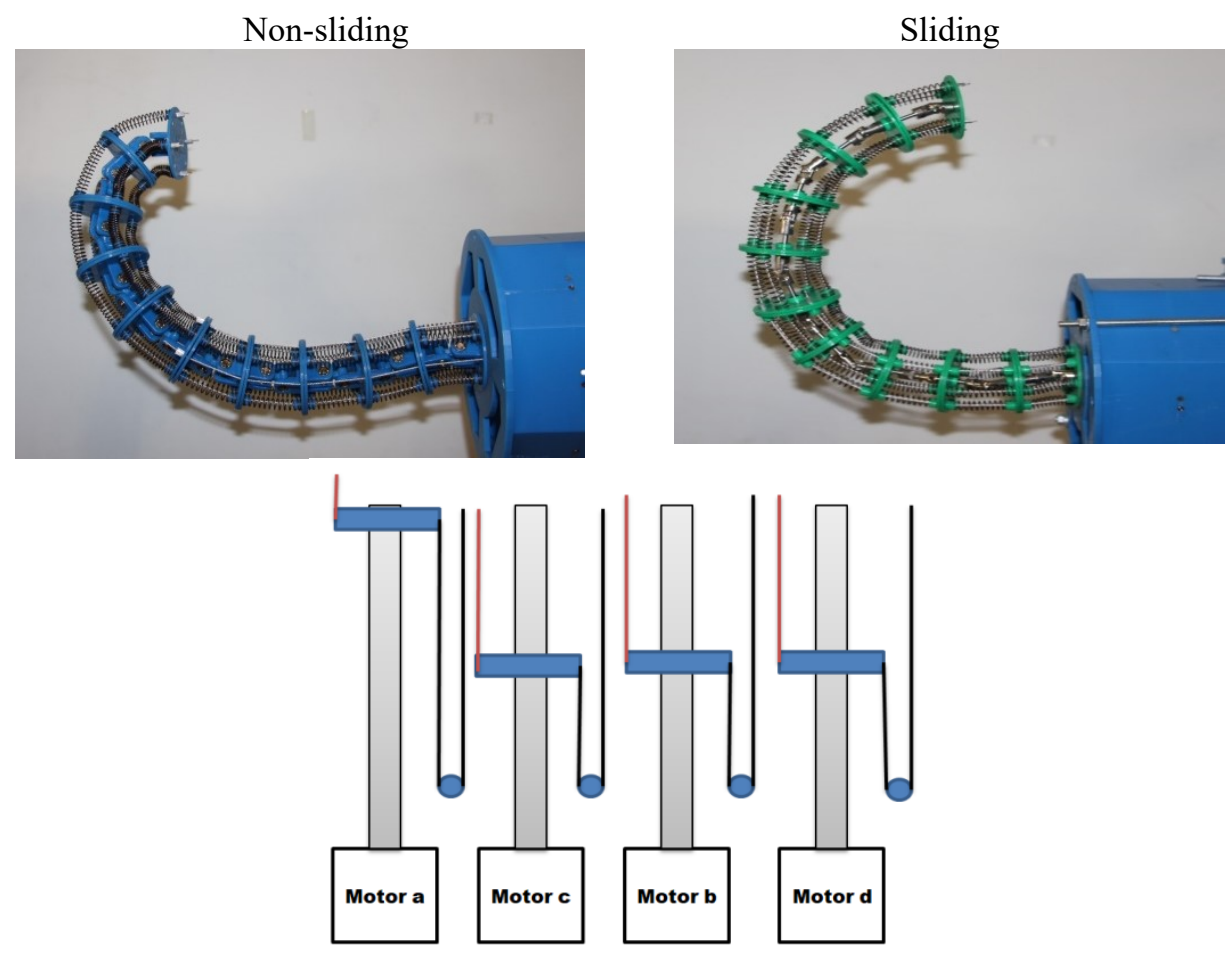

Actuation unit arrangement

Fig.11 Bending comparison between prototype model without sliding discs and the TakoBot with sliding discs
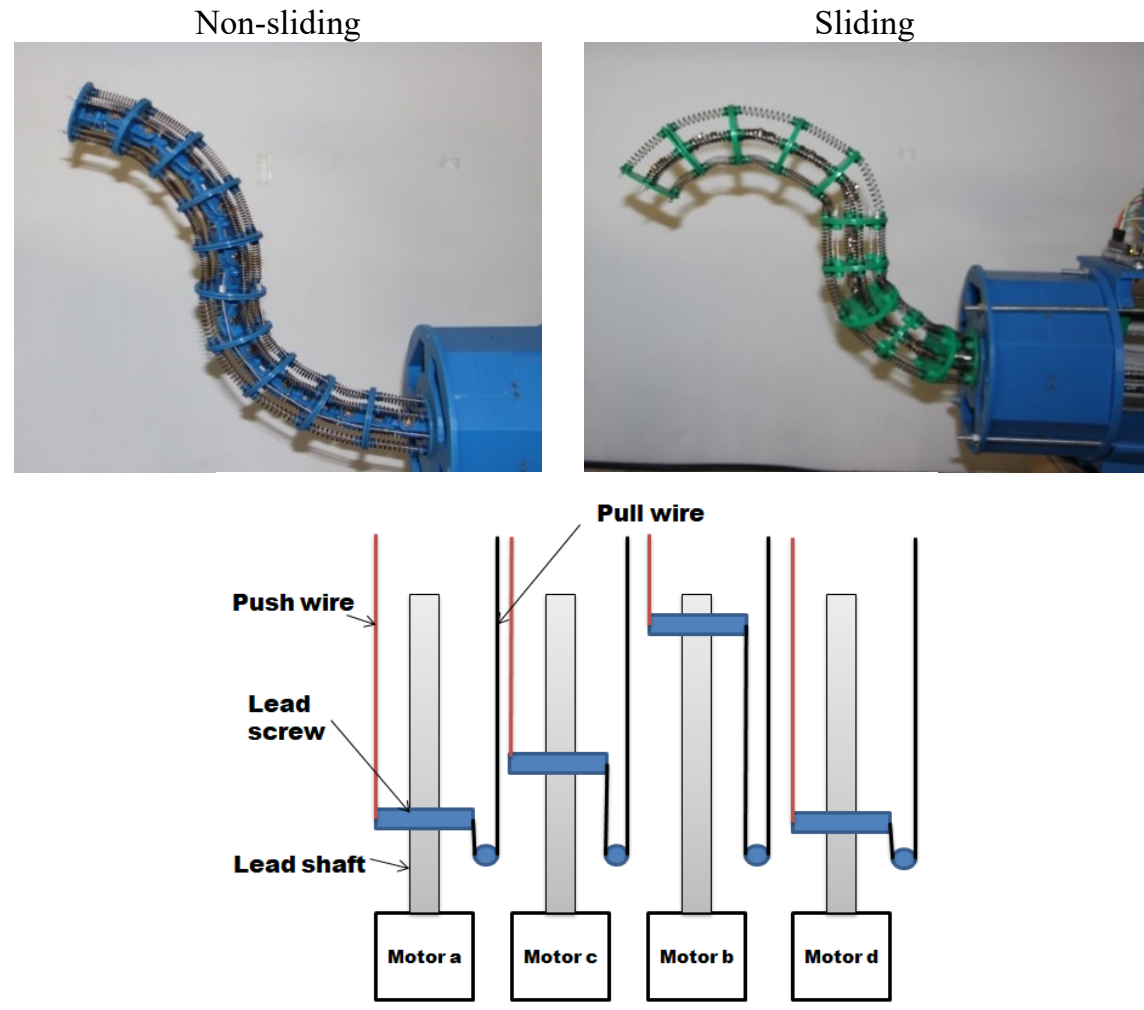

Actuation unit arrangement

Fig.12 "S" shape comparison between prototype model without sliding discs and the TakoBot with sliding discs 
Finally, as evidence of our sliding concept, we measured angles that adjacent discs take. As shown in Fig.13a, the disc positions of TakoBot passively change to balance the static force during bending motions. Therefore, this minimized the bending stress along the backbone, which provides the above mentioned beneficial features. To conduct this experiment we developed a special code only for limited motions.
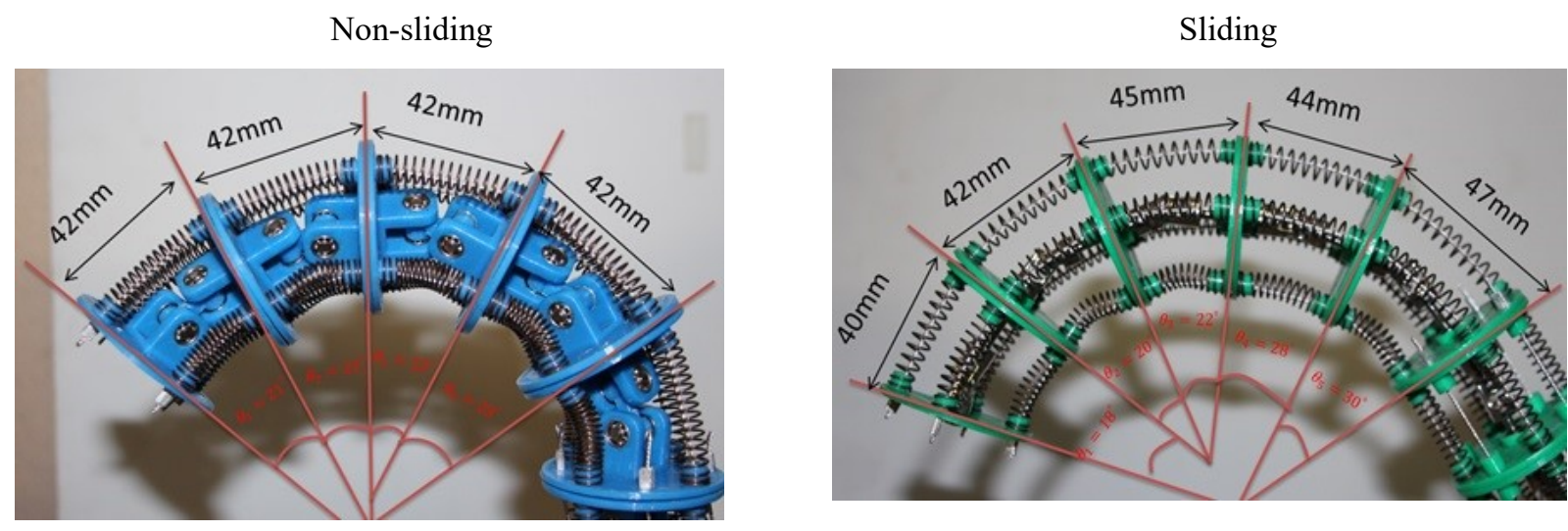

a) The measurement of disc displacement

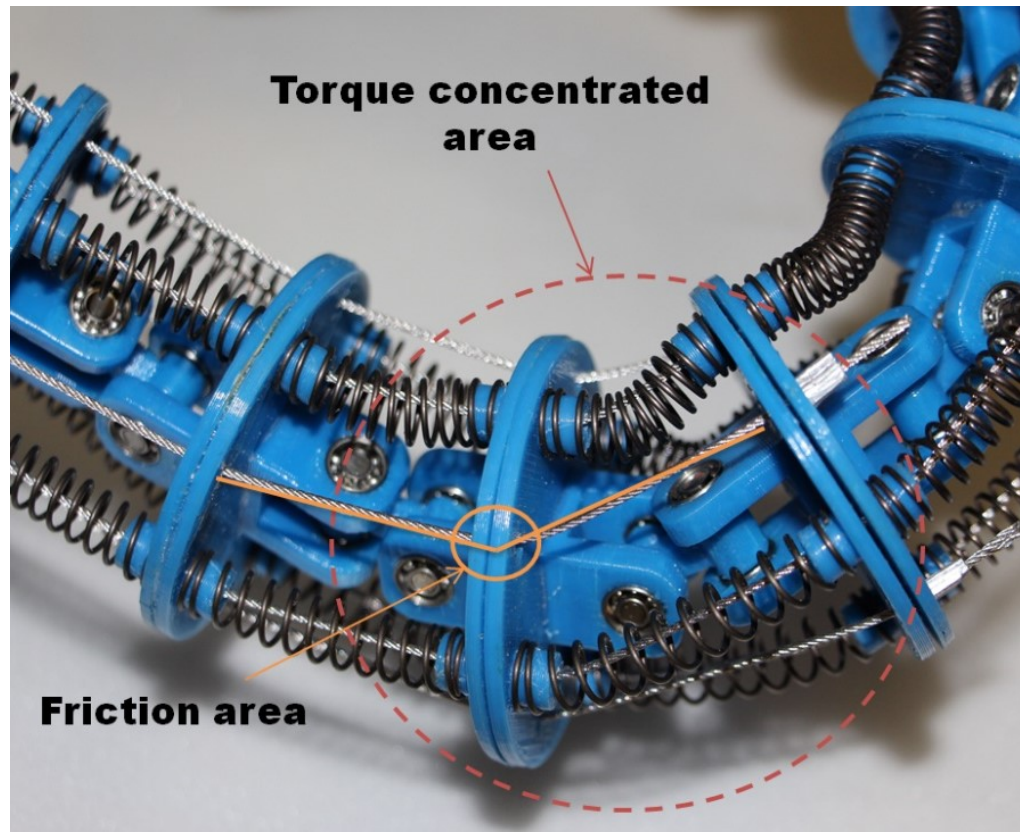

Fig. 13 b) Torque concentrated area in non-sliding prototype

\section{Kinematic and kinetic formulation}

There are some kinematic models for continuum manipulators describing robot structure and design in some ways. The recently published work of Sadati that is a comparative study of five methods of kinematic solutions gives a detailed explanation of models for continuum manipulators [27]. According to this study, most discrete wire-driven continuum robots use an equivalent lumped system model, where the manipulator is a highly articulated rigid link system with a high number of links connected through universal joints or spherical joints bridged by virtual springs and a damper. In our proposed robot, we can also use such modelling to represent the actual kinematic and kinetic structure.

In this chapter, we derive equations representing the force balance between adjacent sliding discs in addition to equations representing the torque balance between adjacent universal joints.

\begin{tabular}{|cl|}
\hline Nomenclature & Definition \\
Symbol & Total number of segments \\
$m$ & Number of segments belonging to the second section \\
$\Sigma_{i}$ & $i$ th coordinate system \\
$H_{\boldsymbol{i}, j}$ & homogeneous transformation matrix from $i$ th coordinate system to $j$ th \\
\hline
\end{tabular}




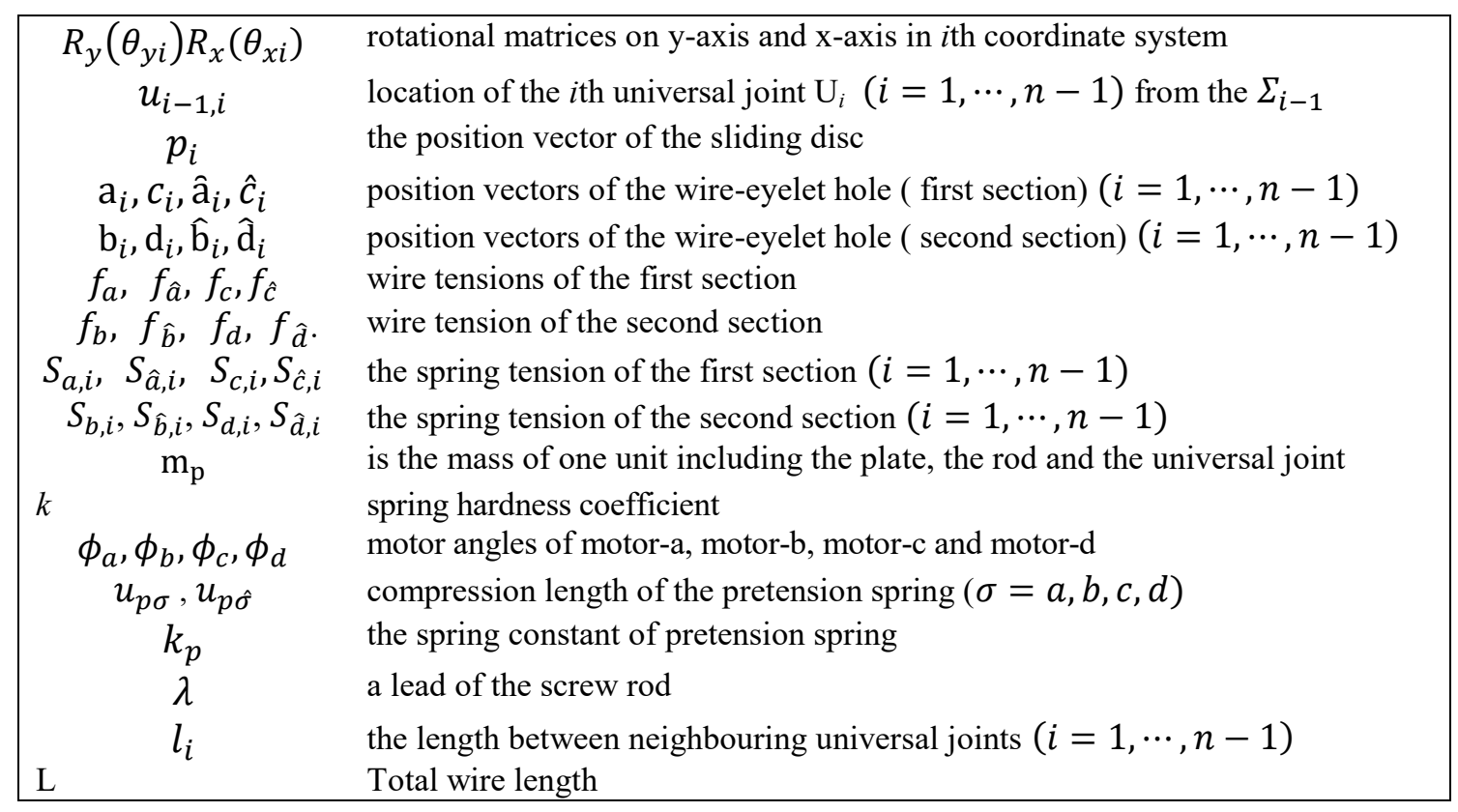

\subsection{Assumptions.}

(1) No friction is assumed that might be occurred between wires and eyelets in discs.

Wires go through the inside springs (see Fig.4), which will avoid any undue bending of the wire at the edge of an eyelet. It will prevent it from generating too much friction.

(2) All discs do not rotate about a backbone shaft.

Discs located at the base and the tip do not rotate (see Fig.2 ), which means that the twisting angle of the slender part is totally zero. Therefore, rotation of any other discs will be considered to be close to zero.

\subsection{Forward kinematics formulation}

Coordinate systems are set at every universal joint

The homogeneous coordinate transform matrices

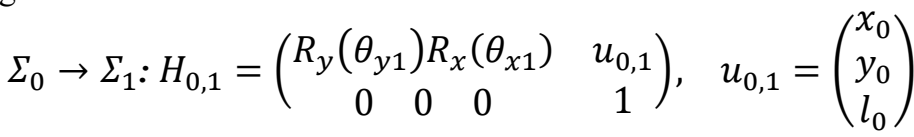

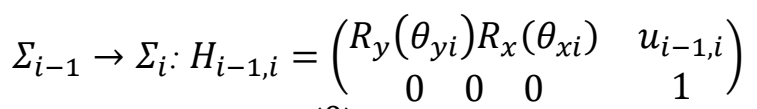

$$
\begin{aligned}
& u_{i-1, i}=\left(\begin{array}{l}
0 \\
0 \\
L
\end{array}\right),(i=2, \cdots, n)
\end{aligned}
$$

where $\left(\begin{array}{lll}x_{0} & y_{0} & l_{0}\end{array}\right)^{T}$ is an initial position of the base. Two rotation matrices have:

$R_{x}\left(\theta_{x i}\right)=\left(\begin{array}{ccc}1 & 0 & 0 \\ 0 & \cos \theta_{x i} & -\sin \theta_{x i} \\ 0 & \sin \theta_{x i} & \cos \theta_{x i}\end{array}\right), \quad R_{y}\left(\theta_{y i}\right)=\left(\begin{array}{ccc}\cos \theta_{y i} & 0 & \sin \theta_{y i} \\ 0 & 1 & 0 \\ -\sin \theta_{y i} & 0 & \cos \theta_{y i}\end{array}\right)$

Multiplying the H-matrices successively, we get unit vectors and the position vector of the $i$ th coordinate system (Fig.14) 


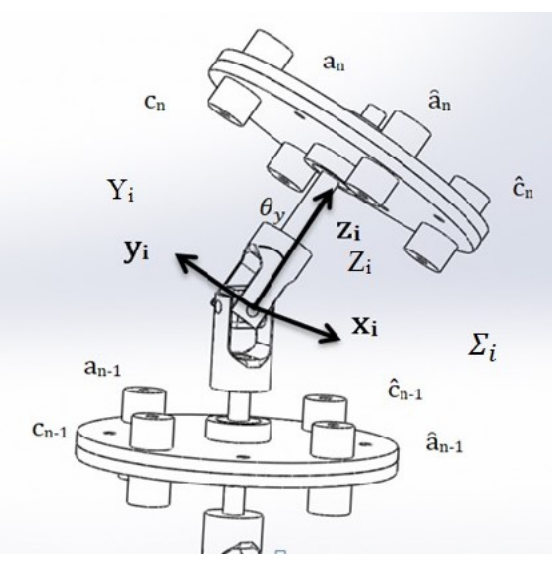

Fig.14 Coordinate system of $i$ th segment

The position vector $p_{i}$ of the end-point $\mathrm{P}_{n}$ and position of sliding plates $\mathrm{P}_{i}(i=1, \cdots, n-1)$ of the manipulator are obtained by (see Fig.15),

$$
\left(\begin{array}{c}
p_{i} \\
1
\end{array}\right)=H_{0, i}\left(\begin{array}{llll}
0 & 0 & l_{i} & 1
\end{array}\right)^{T}, \quad(i=1, \cdots, n)
$$

where $l_{n}$ is a fixed length between the nth universal joint and the tip plate

$$
\begin{gathered}
a_{0}=\left(\begin{array}{c}
a_{x} \\
a_{y} \\
0
\end{array}\right), b_{0}=\left(\begin{array}{c}
b_{x} \\
b_{y} \\
0
\end{array}\right), c_{0}=\left(\begin{array}{c}
c_{x} \\
c_{y} \\
0
\end{array}\right), \quad d_{0}=\left(\begin{array}{c}
d_{x} \\
d_{y} \\
0
\end{array}\right), \\
\hat{a}_{0}=\left(\begin{array}{c}
\hat{a}_{x} \\
\hat{a}_{y} \\
0
\end{array}\right), \quad \hat{b}_{0}=\left(\begin{array}{c}
\hat{b}_{x} \\
\hat{b}_{y} \\
0
\end{array}\right), \quad \hat{c}_{0}=\left(\begin{array}{c}
\hat{c}_{x} \\
\hat{c}_{y} \\
0
\end{array}\right), \quad \hat{d}_{0}=\left(\begin{array}{c}
\hat{d}_{x} \\
\hat{d}_{y} \\
0
\end{array}\right)
\end{gathered}
$$

Position vectors of 4 hole are obtained as

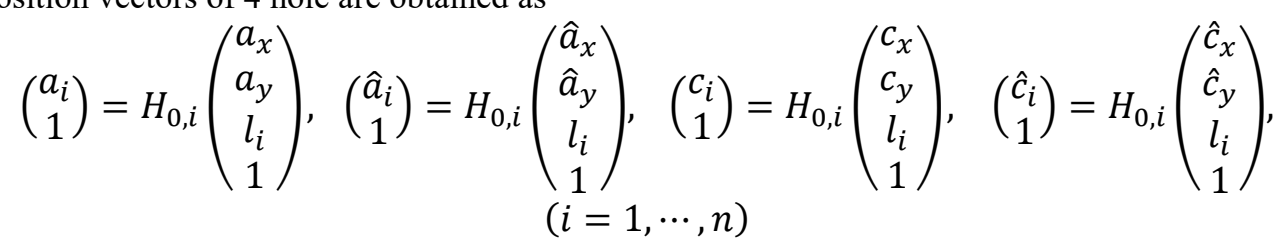

where $l_{i}$ is an axial length between the $i$ th universal joint and the $i$ th plate, which varies as the plate slides along rods, except $l_{n}$.

In the same way, position vectors of 4 -hole $\mathrm{b}, \hat{\mathrm{b}}_{i}, \mathrm{~d}_{i}, \widehat{\mathrm{d}}_{i}$ at the $i$ th plate $(i=1, \cdots, m)$ are obtained as

$$
\begin{array}{r}
\left(\begin{array}{c}
b_{i} \\
1
\end{array}\right)=H_{0, i}\left(\begin{array}{c}
b_{x} \\
b_{y} \\
l_{i} \\
1
\end{array}\right), \quad\left(\begin{array}{c}
\hat{b}_{i} \\
1
\end{array}\right)=H_{0, i}\left(\begin{array}{c}
\hat{b}_{x} \\
\hat{b}_{y} \\
l_{i} \\
1
\end{array}\right),\left(\begin{array}{c}
d_{i} \\
1
\end{array}\right)=H_{0, i}\left(\begin{array}{c}
d_{x} \\
d_{y} \\
l_{i} \\
1
\end{array}\right), \quad\left(\begin{array}{c}
\hat{d}_{i} \\
1
\end{array}\right)=H_{0, i}\left(\begin{array}{c}
\hat{d}_{x} \\
\hat{d}_{y} \\
l_{i} \\
1
\end{array}\right) \\
(i=1, \cdots, m)
\end{array}
$$




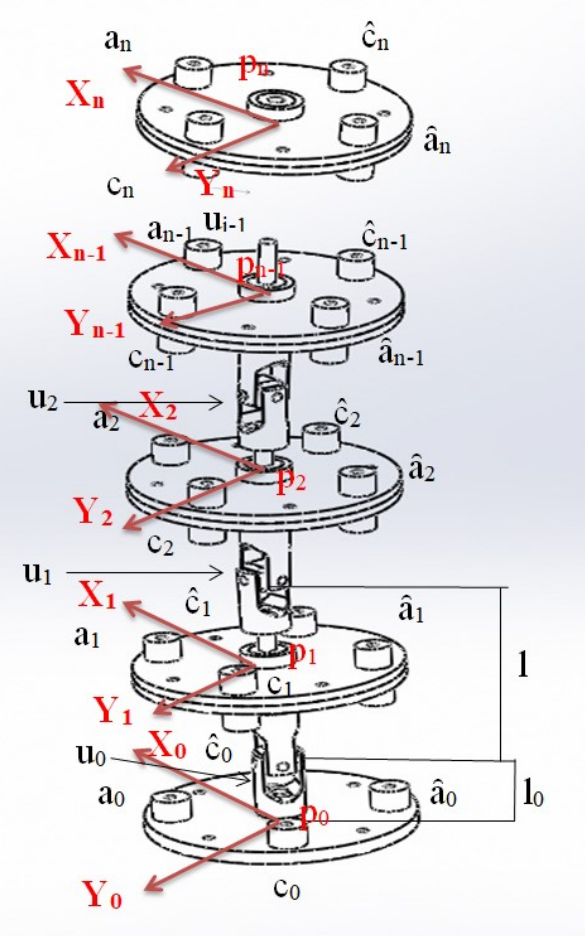

Fig.15 TakoBot kinematic structure

\subsection{Kinetic formulation}

Our continuum manipulator is divided into two sections (see Fig3). The first section is located near the distal part, and the second section is located near the proximal part. The first section is operated by two pairs of two wires for a total of four wires. One pair of wires is controlled by one motor that pulls one wire and pushes the other wire by the same length by using the pulley. While the second section is operated by four pairs of wires for a total of eight wires. Therefore, the second section is controlled by four motors (see Figure 11).

The second section has $m$ units and the first section has $n-m$ units. Four pairs of wires are labelled by $a$ and $\hat{a}, b$ and $\hat{b}, c$ and $\hat{c}$, d and $\hat{d}$,

Equilibrium in moments at $U_{\mathrm{n}}$ belonging to the first section is,

$$
\begin{gathered}
\left(S_{a, n}-f_{a}\right)\left(\overline{a_{n}-a_{n-1}}\right) \times\left(a_{n}-u_{n}\right)+\left(S_{\hat{a}, n}-f_{\hat{a}}\right)\left(\overline{\hat{a}_{n}-\hat{a}_{n-1}}\right) \times\left(\hat{a}_{n}-u_{n}\right) \\
\quad+\left(S_{c, n}-f_{c}\right)\left(\overline{c_{n}-c_{n-1}}\right) \times\left(c_{n}-u_{n}\right)+\left(S_{\hat{c}, n}-f_{\hat{c}}\right)\left(\overline{\hat{c}_{n}-\hat{c}_{n-1}}\right) \\
\quad \times\left(\hat{c}_{n}-u_{n}\right)+m_{w}\left(p_{n}-u_{n}\right) \times g=\left(\begin{array}{lll}
0 & 0 & 0
\end{array}\right)^{T}
\end{gathered}
$$

where $\overline{a_{n}-a_{n-1}}==\left(a_{n}-a_{n-1}\right) /\left|a_{n}-a_{n-1}\right|$, etc. $m_{w}$ is a payload applying at the end-point and $g$ is the gravity acceleration vector. 


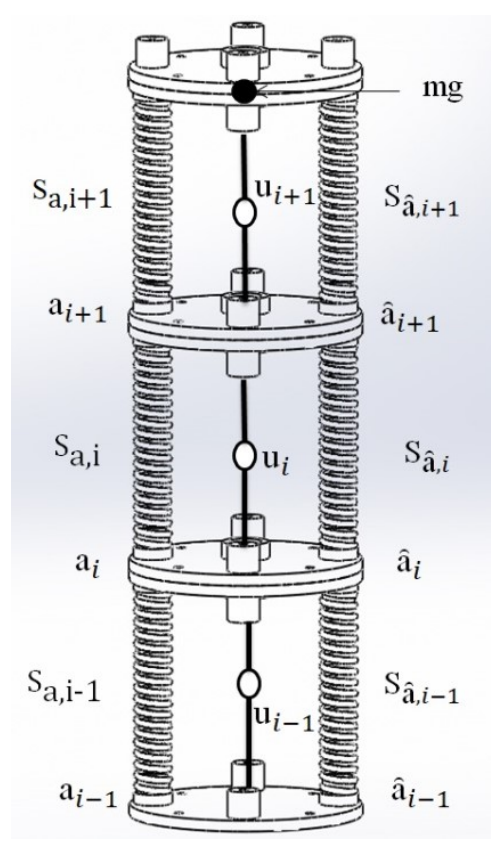

a) First section

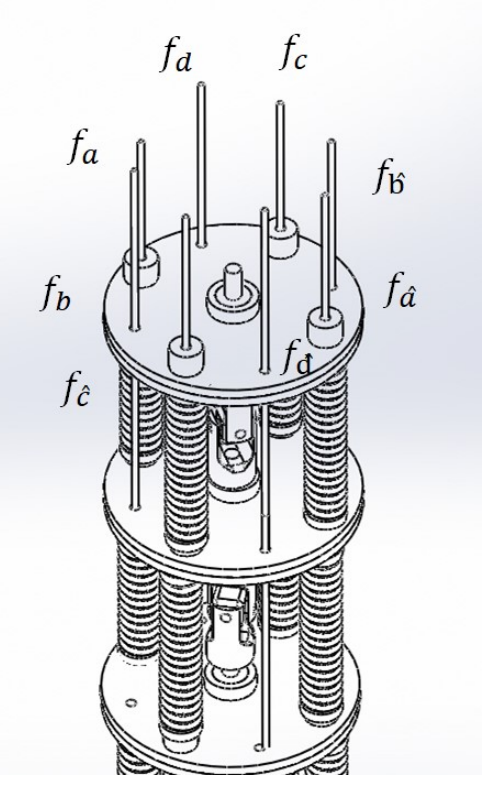

b) Second section

Fig.16 TakoBot kinetic structure

Equilibrium in moments at $\mathrm{U}_{i},(i=m+1, \cdots, n-1)$, belonging to the first section is where $f_{a}, f_{\hat{a}}, f_{c}, f_{\hat{c}}$ are wire tensions (Fig 16a), $S_{a, i}, S_{\hat{a}, i}, S_{c, i}, S_{\hat{c}, i},(i=m+1, \cdots, n)$ are spring tensions of the $i$ th unit. " $X$ " means a cross product $m_{p}$ is the mass of one unit including the plate, the rod and the universal joint.

The spring tensions are obtained as,

$$
\begin{aligned}
& \left(-S_{a, i+1}\left(\overline{a_{i+1}-a_{i}}\right)+S_{a, i}\left(\overline{a_{i}-a_{i-1}}\right)\right) \times\left(a_{i}-u_{i}\right)+\left(-S_{\hat{a}, i+1}\left(\overline{\hat{a}_{i+1}-\hat{a}_{i}}\right)+\right. \\
& \left.S_{\hat{a}, i}\left(\overline{\hat{a}_{i}-\hat{a}_{i-1}}\right)\right) \times\left(\hat{a}_{i}-u_{i}\right)+\left(-S_{c, i+1}\left(\overline{c_{i+1}-c_{i}}\right)+S_{c, i}\left(\overline{c_{i}-c_{i-1}}\right)\right) \times\left(c_{i}-u_{i}\right)+ \\
& \left(-S_{\hat{c}, i+1}\left(\overline{\hat{c}_{i+1}-\hat{c}_{i}}\right)+S_{\hat{c}, i}\left(\overline{\hat{c}_{i}-\hat{c}_{i-1}}\right)\right) \times\left(\hat{c}_{i}-u_{i}\right)+\left(m_{w}\left(p_{n}-u_{i}\right)+m_{p} \sum_{k=i+1}^{n-1}\left(p_{k}-\right.\right. \\
& \left.\left.u_{i}\right)\right) \times g=\left(\begin{array}{lll}
0 & 0 & 0
\end{array}\right)^{T} \\
& S_{a, i}=k\left(L-\left|a_{i}-a_{i-1}\right|\right), S_{\hat{a}, i}=k\left(L-\left|\hat{a}_{i}-\hat{a}_{i-1}\right|\right), \\
& S_{c, i}=k\left(L-\left|c_{i}-c_{i-1}\right|\right), \quad S_{\hat{c}, i}=k\left(L-\left|\hat{c}_{i}-\hat{c}_{i-1}\right|\right),
\end{aligned}
$$

with the spring coefficient $k$. Equations (8) and (9) contain 3(n-m) equations including 4(n-m)-1 variables of the $n-m$ universal joints angles $\theta_{x i}, \theta_{y i}, \theta_{z i},(i=m+1, \cdots, n)$ and slide length of plates $l_{i}(i=$ $m+1, \cdots, n-1)$.

Equilibrium in force at $i$ th plate $(i=m+1, \cdots, n-1)$ is,

$$
\begin{aligned}
& \left(-S_{a, i+1}\left(\overline{a_{i+1}-a_{i}}\right)+S_{a, i}\left(\overline{a_{i}-a_{i-1}}\right)-S_{\hat{a}, i+1}\left(\overline{\hat{a}_{i+1}-\hat{a}_{i}}\right)\right. \\
& +S_{\hat{a}, i}\left(\overline{\hat{a}_{i}-\hat{a}_{i-1}}\right)-S_{c, i+1}\left(\overline{c_{i+1}-c_{i}}\right)+S_{c, i}\left(\overline{c_{i}-c_{i-1}}\right)-S_{\hat{c}, i+1}\left(\overline{\hat{c}_{i+1}-\hat{c}_{i}}\right) \\
& \left.+S_{\hat{c}, i}\left(\hat{c}_{i}-\hat{c}_{i-1}\right)+(n-i) m_{p} g\right) \cdot\left(p_{i}-u_{i}\right)=0
\end{aligned}
$$

Equation (11) provides $n-m-1$ equations. Combined it with (8) and (9), we obtain 4(n-m)-1 equations, which suffices in number to solve for $4(n-m)-1$ variables; $\theta_{x, i}, \theta_{y, i}, \theta_{z, i}(i=m+$ $1, \cdots, n)$ and $l_{i}(i=m+1, \cdots, n-1)$ for a given set of wire tensions $f_{a}, f_{\hat{a}}, f_{c}, f_{\hat{c}}$. 
Equilibrium in moments at $\mathrm{U}_{m}$, the universal joint located at the most distal position belonging to the second section is

$$
\begin{gathered}
-S_{a, m+1}\left(\overline{a_{m+1}-a_{m}}\right) \times\left(a_{m}-u_{m}\right)+\left(S_{b, m}-f_{b}\right)\left(\overline{b_{m}-b_{m-1}}\right) \times\left(b_{m}-u_{m}\right) \\
-S_{\hat{a}, m+1}\left(\overline{\hat{a}_{m+1}-\hat{a}_{m}}\right) \times\left(\hat{a}_{m}-u_{m}\right)+\left(S_{\hat{b}, m}-f_{\hat{b}}\right)\left(\overline{\hat{b}_{m}-\hat{b}_{m-1}}\right) \times\left(\hat{b}_{m}-u_{m}\right) \\
-S_{c, m+1}\left(\overline{c_{m+1}-c_{m}}\right) \times\left(c_{m}-u_{m}\right)+\left(S_{d, m}-f_{d}\right)\left(\overline{d_{m}-d_{m-1}}\right) \times\left(d_{m}-u_{m}\right) \\
-S_{\hat{c}, m+1}\left(\overline{\hat{c}_{m+1}-\hat{c}_{m}}\right) \times\left(\hat{c}_{m}-u_{m}\right)+\left(S_{\hat{d}, m}-f_{\hat{d}}\right)\left(\overline{\left.\hat{d}_{m}-\hat{d}_{m-1}\right) \times\left(\hat{d}_{m}-u_{m}\right)}\right. \\
+\left(m_{w}\left(p_{n}-u_{m}\right)+m_{p} \sum_{k=m+1}^{n-1}\left(p_{k}-u_{m}\right)\right) \times g=\left(\begin{array}{lll}
0 & 0 & 0
\end{array}\right)^{T}
\end{gathered}
$$

For the second segment, we can derive similar equations as (9), (10) and (11) by replacing $\left\{a_{i}, \hat{a}_{i}, c_{i}, \hat{c}_{i}\right\}$ with $\left\{b_{i}, \hat{b}_{i}, d_{i}, \hat{d}_{i}\right\},\left\{S_{a, i}, S_{\hat{a}, i}, S_{c, i}, S_{\hat{c}, i}\right\}$ with $\left\{S_{b, i}, S_{\hat{b}, i}, S_{d, i}, S_{\hat{d}, i}\right\}$ for $i=$ $1, \cdots, m-1$ in (10) and for $i=1, \cdots, m$ in (10) and (11) (see Fig 16b).

As a result, we obtain $4 m$ equations included by Equation (12), which suffices in number to solve for $4 m$ variables; $\theta_{x, i}, \theta_{y, i}, \theta_{z, i}$ and $l_{i}(i=1, \cdots, m)$ for a given set of wire tensions $f_{b}, f_{\hat{b}}, f_{d}, f_{\hat{d}}$.

Wire tensions $f_{a}, f_{\hat{a}}, f_{c}, f_{\hat{c}}, f_{b}, f_{\hat{b}}, f_{d}, f_{\hat{d}}$ are determined according to 4 motors' angles $\phi_{a}, \phi_{b}, \phi_{c}, \phi_{d}$ (Fig 17)

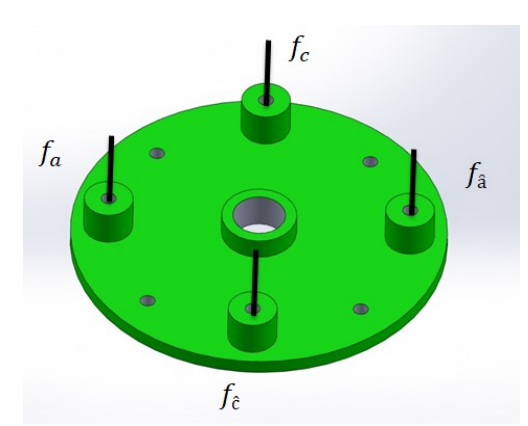

First section spacer disc

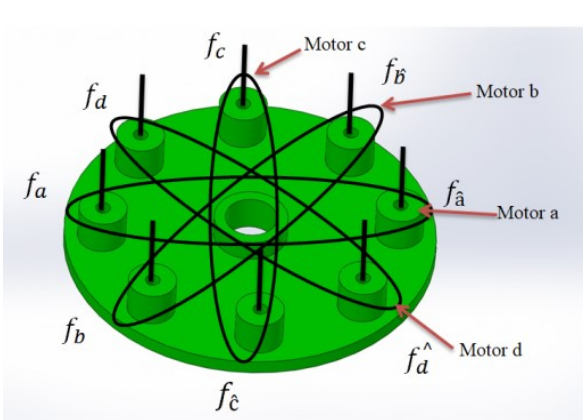

Second section spacer disc

Fig.17 Wire eyelet arrangement on the disc

\subsection{Pretention Mechanism Formulation}

Fig.18 shows a model of pretension mechanism (see Fig.5).

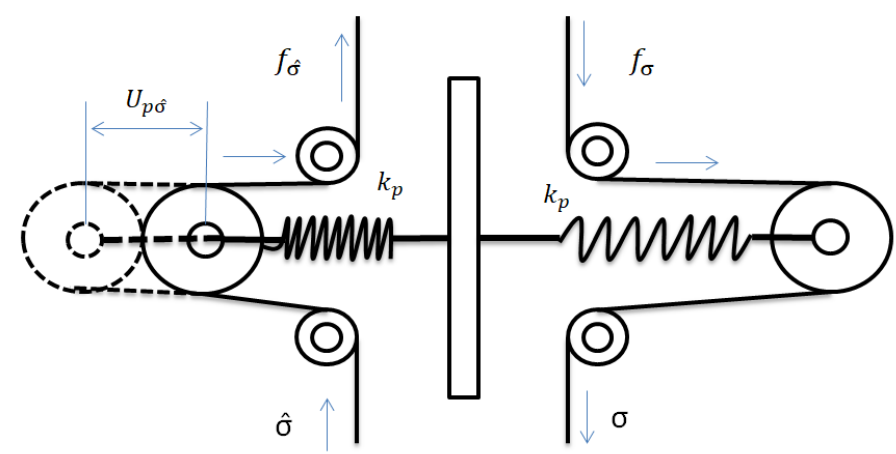

Fig.18 Pretension mechanism nomenclature

The pretension spring receives $2 f$, therefore:

$$
2 f_{\sigma}=k_{p} u_{p \sigma},(\sigma=a, b, c, d), \quad 2 f_{\hat{\sigma}}=k_{p} u_{p \hat{\sigma}},(\hat{\sigma}=\hat{a}, \hat{b}, \hat{c}, \hat{d})
$$

Where $u_{p \sigma}$ and $u_{p \hat{\sigma}}$ are determined by motor rotation angle and wire length 


$$
2 u_{p \sigma}=2 \bar{u}_{p \sigma}+\frac{\lambda \varphi_{\sigma}}{2 \pi}+\sum_{i=1}^{n}\left|\sigma_{i}-\sigma_{i-1}\right|-n L, \quad 2 u_{p \hat{\sigma}}=2 \bar{u}_{p \hat{\sigma}}-\frac{\lambda \varphi_{\sigma}}{2 \pi}+
$$

where $2 \bar{u}_{p \sigma}$ and $2 \bar{u}_{p \hat{\sigma}}$ are compression length of the pretension spring, which are preset initially.

Substituting Equation (14) into Equation (13), we have;

$$
\begin{array}{r}
f_{\sigma}=\frac{1}{2} k_{p}\left(2 \bar{u}_{p \sigma}+\frac{\lambda \varphi_{\sigma}}{4 \pi}+\frac{1}{2}\left(\sum_{i=1}^{n}\left|\sigma_{i}-\sigma_{i-1}\right|-n L\right)\right), f_{\hat{\sigma}}=\frac{1}{2} k_{p}\left(2 \bar{u}_{p \hat{\sigma}}-\frac{\lambda \varphi_{\sigma}}{4 \pi}+\frac{1}{2}\left(\sum_{i=1}^{n}\left|\hat{\sigma}_{i}-\hat{\sigma}_{i-1}\right|-n L\right)\right) \\
f_{a}=\frac{1}{2} k_{p}\left(2 \overline{\mathrm{U}}_{p a}+\frac{\lambda \phi_{a}}{4 \pi}+\frac{1}{2}\left(\sum_{i=1}^{n}\left|a_{i}-a_{i-1}\right|-n L\right)\right), f_{\hat{a}}=\frac{1}{2} k_{p}\left(2 \overline{\mathrm{U}}_{p \hat{\mathrm{a}}}-\frac{\lambda \phi_{a}}{4 \pi}+\frac{1}{2}\left(\sum_{i=1}^{n}\left|\hat{a}_{i}-\hat{a}_{i-1}\right|-n L\right)\right) \\
f_{c}=\frac{1}{2} k_{p}\left(2 \overline{\mathrm{U}}_{p c}+\frac{\lambda \phi_{c}}{4 \pi}+\frac{1}{2}\left(\sum_{i=1}^{n}\left|c_{i}-c_{i-1}\right|-n L\right)\right), f_{\hat{\mathrm{c}}}=\frac{1}{2} k_{p}\left(2 \overline{\mathrm{U}}_{p \hat{\mathrm{c}}}-\frac{\lambda \phi_{\hat{\mathrm{c}}}}{4 \pi}+\frac{1}{2}\left(\sum_{i=1}^{n}\left|\hat{\mathrm{c}}_{i}-\hat{\mathrm{c}}_{i-1}\right|-n L\right)\right) \\
f_{b}=\frac{1}{2} k_{p}\left(2 \overline{\mathrm{U}}_{p b}+\frac{\lambda \phi_{b}}{4 \pi}+\frac{1}{2}\left(\sum_{i=1}^{n}\left|b_{i}-b_{i-1}\right|-n L\right)\right), f_{\hat{b}}=\frac{1}{2} k_{p}\left(2 \overline{\mathrm{U}}_{p \hat{b}}-\frac{\lambda \phi_{\hat{b}}}{4 \pi}+\frac{1}{2}\left(\sum_{i=1}^{n}\left|\hat{b}_{i}-\hat{b}_{i-1}\right|-n L\right)\right) \\
f_{d}=\frac{1}{2} k_{p}\left(2 \overline{\mathrm{U}}_{p d}+\frac{\lambda \phi_{d}}{4 \pi}+\frac{1}{2}\left(\sum_{i=1}^{n}\left|d_{i}-d_{i-1}\right|-n L\right)\right), f_{d}=\frac{1}{2} k_{p}\left(2 \overline{\mathrm{U}}_{p d}-\frac{\lambda \phi_{d}}{4 \pi}+\frac{1}{2}\left(\sum_{i=1}^{n}\left|\hat{d}_{i}-d_{i-1}\right|-n L\right)\right)
\end{array}
$$

\subsection{Formulation of inverse solution}

According to the given set of variables $\theta_{x, i}, \theta_{y, i}, \theta_{z, i}(i=1, \cdots, n)$ and $l_{i}(i=$ $1, \cdots, n-1)$, we calculate the end-point position by Equation (4),

$$
\left(\begin{array}{c}
p_{n} \\
1
\end{array}\right)=H_{0, n}\left(\begin{array}{c}
0 \\
0 \\
l_{n} \\
1
\end{array}\right)=\left(\begin{array}{cccc}
i_{n} & j_{n} & k_{n} & r_{n} \\
0 & 0 & 0 & 1
\end{array}\right)\left(\begin{array}{c}
0 \\
0 \\
l_{n} \\
1
\end{array}\right)=\left(\begin{array}{c}
k_{n} l_{n}+r_{n} \\
1
\end{array}\right)
$$

Taking a total differentiation of $p_{n}=k_{n} l_{n}+r_{n}$ with respect to $\theta_{x, i}, \theta_{y, i}, \theta_{z, i}(i=$ $1, \cdots, n)$ and $l_{i}(i=1, \cdots, n-1)$ and also motor angles $\phi_{a}, \phi_{b}, \phi_{c}, \phi_{d}$,

$$
\Delta p_{n}=\frac{\partial p_{n}}{\partial v} \Delta v+\frac{\partial p_{n}}{\partial \phi} \Delta \phi
$$

where, $\boldsymbol{v}=\left(\theta_{x 1}, \theta_{x 2}, \cdots, \theta_{x n}, \theta_{y 1}, \theta_{y 2}, \cdots, \theta_{y n} \cdots, l_{1}, l_{2}, \cdots, l_{n-1}\right) \in \mathcal{R}^{4 n-1}$ and $\phi=\left(\phi_{a}, \phi_{b}, \phi_{c}, \phi_{d}\right) . \frac{\partial p_{n}}{\partial v} \in \mathcal{R}^{3 \times 4 n-1}$ and $\frac{\partial p_{n}}{\partial \phi} \in \mathcal{R}^{3 \times 4}$.

Let $w=\left(w_{1}, w_{2}, \cdots w_{4 n-1}\right)^{T}=0_{4 n-1}$ represents the $4 n-1$ equations provided by Equations (8-11), which also includes $\theta_{x, i}, \theta_{y, i},(i=1, \cdots, n), l_{i}(i=1, \cdots, n-1)$ and also motor angles $\phi_{a}, \phi_{b}, \phi_{c}, \phi_{d}$.

Taking a total differentiation for $w=\left(w_{1}, w_{2}, \cdots w_{4 n}\right)=0_{4 n-1}$ as well, we have,

$$
\Delta w=\frac{\partial w}{\partial v} \Delta v+\frac{\partial w}{\partial \phi} \Delta \phi=0_{4 n-1}
$$

where $\frac{\partial w}{\partial v} \in \mathcal{R}^{(4 n-1) \times(4 n-1)}$ and $\frac{\partial w}{\partial \phi} \in \mathcal{R}^{(4 n-1) \times 4}$. Since $\frac{\partial w}{\partial v}$ is a square matrix, we can solve Equation (20) with respect to the vector $\Delta v$ as,

$$
\Delta v=-\left(\frac{\partial w}{\partial v}\right)^{-1} \frac{\partial w}{\partial \phi} \Delta \phi
$$

Substituting Equation (17) into Equation (15), we have

$$
\Delta p_{n}=-\frac{\partial p_{n}}{\partial v}\left(\frac{\partial w}{\partial v}\right)^{-1} \frac{\partial w}{\partial \phi} \Delta \phi+\frac{\partial p_{n}}{\partial \phi} \Delta \phi=\left(\frac{\partial p_{n}}{\partial \phi}-\frac{\partial p_{n}}{\partial v}\left(\frac{\partial w}{\partial v}\right)^{-1} \frac{\partial w}{\partial \phi}\right) \Delta \phi=J \Delta \phi
$$


which can be solved for $\Delta \phi$, by using a generalized inverse of the Jacobian $J \in \mathcal{R}^{3 \times 4}$

$$
\Delta \phi=J^{\dagger} \Delta p_{n}+P^{\perp}(J) \Delta \phi_{N}
$$

where $J^{\dagger} \in \mathcal{R}^{4 \times 3}$ is a generalized inverse of $J$ and $P^{\perp}(J) \in \mathcal{R}^{4 \times 4}$ is a null projection operator of $J$, and $\Delta \phi_{N} \in \mathcal{R}^{4}$ is a correction of $\phi$ so as to minimize a positive scalar potential $\varphi$ by making use of a redundant actuation. We use $J^{\dagger}=J^{T}\left(J J^{T}\right)^{-1}$ and $P^{\perp}(J)=I-J^{\dagger} J$.

Equation (19) provides a variation of motor angles $\Delta \phi$ for a given position and direction variation $\Delta p_{n}$

Applying the Euler method, we have the following variety equation

$$
\varphi+(\partial \varphi / \partial \phi) \Delta \phi_{\mathrm{N}}=0
$$

which is solved by

$$
\Delta \phi_{\mathrm{N}}=-\frac{\varphi}{(\partial \varphi / \partial \phi)(\partial \varphi / \partial \phi)^{\mathrm{T}}}\left(\frac{\partial \varphi}{\partial \phi}\right)^{\mathrm{T}}
$$

As a candidate, we take $k_{n z}^{2}$, where, $k_{n z}^{2}$ is the $\mathrm{z}$ component of $k_{n}$ : The unit vector of the end-point orienting an axial direction. It means that the axial direction and the end-point takes on a horizontal plain as far as possible while keeping a designated position (see Fig 19).

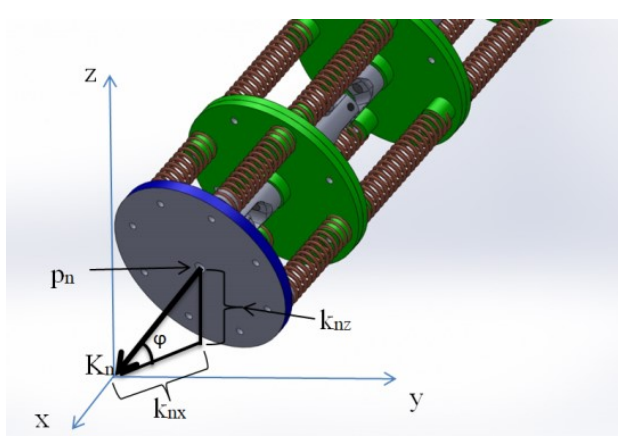

Fig. 19 Orientation of the end-effector

\section{Simulation studies}

The simulation studies were done for the following purposes.

(1) To validate the formulations described in the former section.

(2) To validate the effect of disc sliding by comparing cases of sliding dises and non-sliding discs.

Due to the limitations of computer memory capacity, we confined the number of segments to 4 in the first section and 3 in the second section (the actual Takobot has 4 and 5, respectively). Therefore, the total number of segments $n$ is 7 and total number of equations $3 n-1$ is 20 . Table 2 shows the physical parameters used in the simulation.

Table 2. Values used for the simulation

\begin{tabular}{|l|l|l|}
\hline $\mathrm{Ls}$ & Natural length of spring & $60[\mathrm{~mm}]$ \\
\hline $\mathrm{ks}$ & Spring constant of the springs between UJs & $0.8[\mathrm{~N} / \mathrm{mm}]$ \\
\hline $\mathrm{kp}$ & Spring constant of the pretension springs & $1.6[\mathrm{~N} / \mathrm{mm}]$ \\
\hline $\mathrm{m}_{\mathrm{w}}$ & Payload & $0[\mathrm{~kg}]$ \\
\hline $\mathrm{lp}$ & Pulling length to yield pretension & $30[\mathrm{~mm}]$ \\
\hline $\mathrm{gz}$ & Gravity acceleration & $9.80665\left[\mathrm{~mm} / \mathrm{s}^{\wedge} 2\right]$ \\
\hline
\end{tabular}

\subsection{Forward motions}




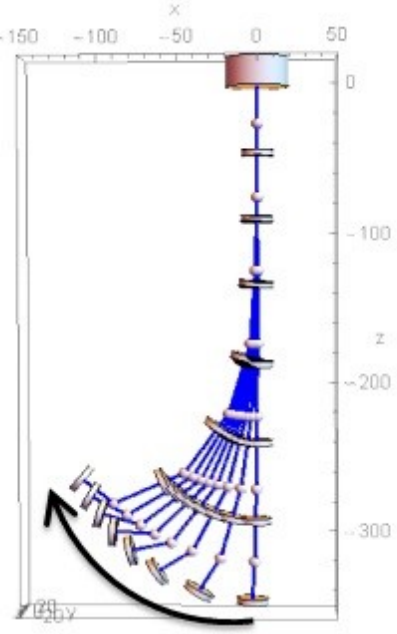

(a) the case of sliding discs

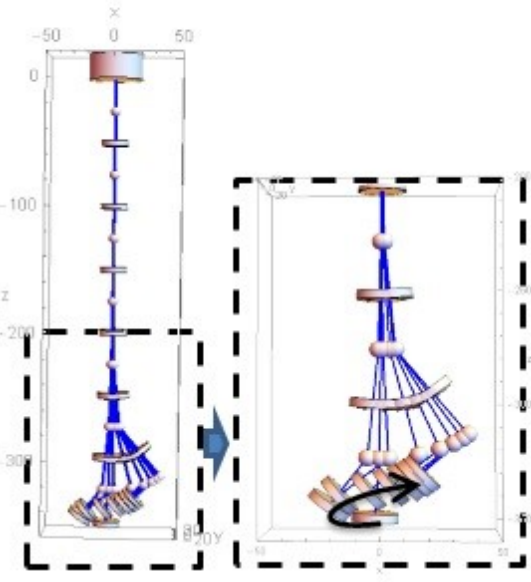

(b) the case of non-sliding discs

Fig.20 Simulation results when only the motor-a rotates 6 revolution

It observes the motion of the TakoBot when only motor-a rotates $12 \pi[\mathrm{rad}]$ ( 6 [rev]) with a constant rate. It aims to compare motions between the disc sliding and non-sliding. Newton's method was employed to converge 20 equations close to be zero with the specified motor angles. Fig.20 shows the motion of the TakoBot. Small spheres represent universal joints. The springs located between adjacent discs are not drawn. As shown in Fig.20, the end-plate takes a monotonically movement with the monotonically rotation of the motor-a in the case of disc sliding. It's desirable for control. However, the end-plate takes back and forth motion with the same rotation of motor-a in the case of non-sliding disc. It is due to a high-bending torque yielded at the last universal joint. This non-monotonically response will make the control difficult. In the case of real application (Fig. 11), applied motor torque concentrates on the bending joint which causes more friction interference between the wire and spacer disc eyelets. Thus, the non-sliding prototype consumes more power as well.
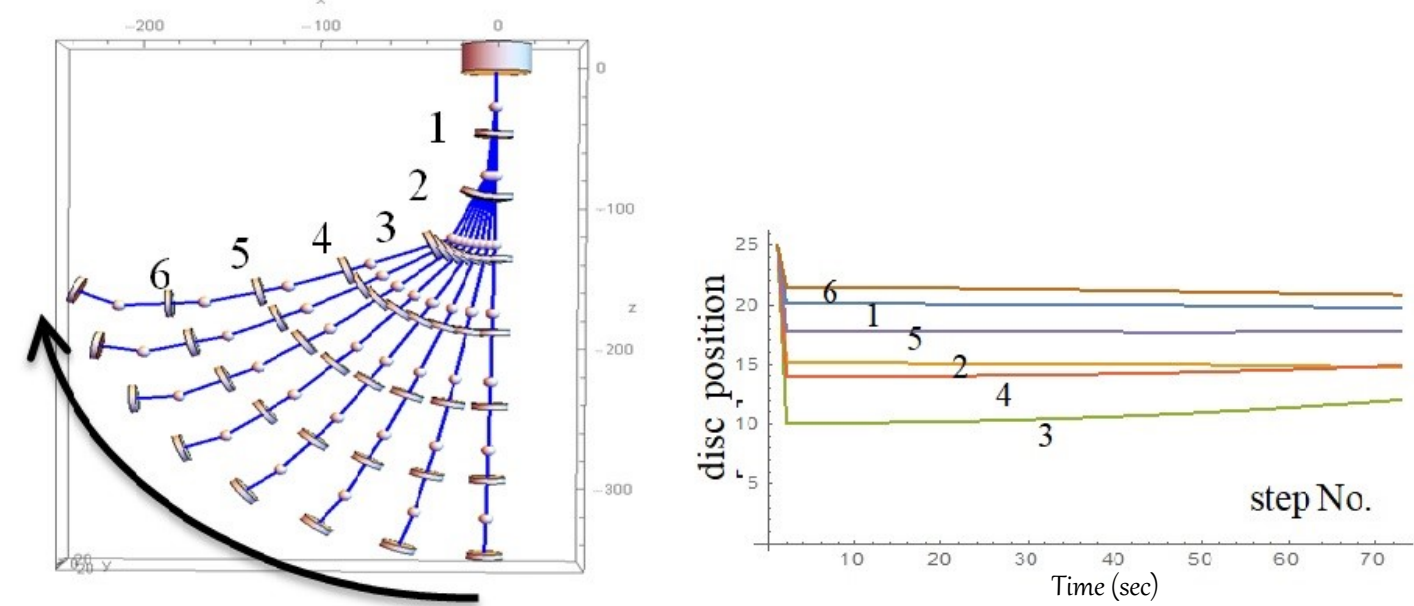

(a) Motion of TakoBot

(b) Positions of discs (length from the former UJ)

Fig.21 Simulation results when the motor-a, and motor-c rotates 6 rotations and the motor-d rotates -6 revolutions simultaneously

Fig.21 (a) shows the motion of the TacoBot when motor-a and motor-c rotate $12 \pi[\mathrm{rad}]$ (6 [rev]) and motor-d rotates $-12 \pi[\mathrm{rad}](-6[\mathrm{rev}])$ at a constant rate. Fig.21(b) shows the sliding length of the discs during the motion. The initial positions of all discs are 25 [mm]. At the first, all of them slide largely because the specified pre-tension is assumed to be loaded at the first step. 


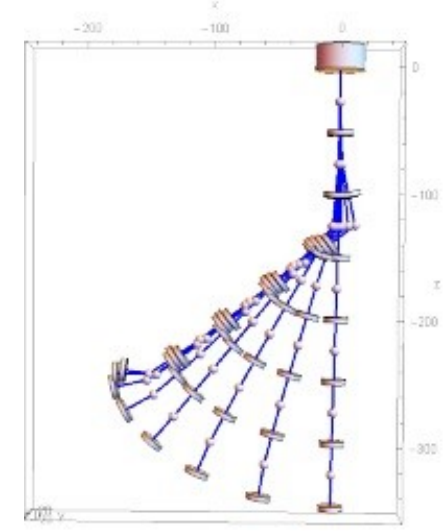

Fig.22 Simulation results for the case of non-sliding discs when motor-a, and motor-c rotates 6 rotations and motor-d rotates -6 revolutions simultaneously

Fig. 22 shows the motion of the prototype with non-sliding discs when the motors rotate in the same way as shown in Fig.21. As shown, the tip of the manipulator lifts up less than the case of sliding discs. This phenomenon is the same as those observed in the actual experiment shown in Fig.9.

\subsection{Control motions}

Moreover, to validate the formulation of control, we created the following task: move the endeffector disc $50 \mathrm{~mm}$ along the z-axis and x-axis by 50 steps (Fig 23, 24) (1 mm at every step). Eq.(21) without null-projection term is used. To visualize the motion of the manipulator, we used three motors to get a visual result on a two-dimensional plane (Y-Z). Motion on the other planes would be difficult to identify and verify. TakoBot's four motors are labelled as motor a, motor b, motor c and motor d. The first two motors are for actuating the first section, and the other two motors are for the second section actuation. In this simulation, we actuated motor $\mathrm{a}$, motor $\mathrm{b}$ and motor $\mathrm{d}$. Motor $\mathrm{c}$ mostly remains at zero. This is for manipulator motion on Y-Z plane.

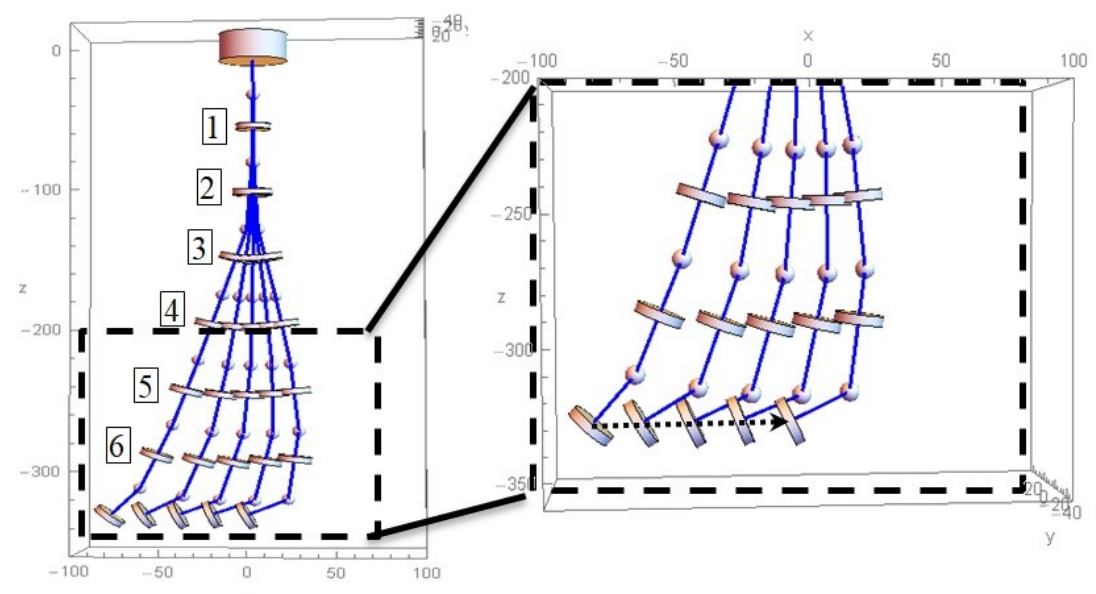

Main view

Zoom view

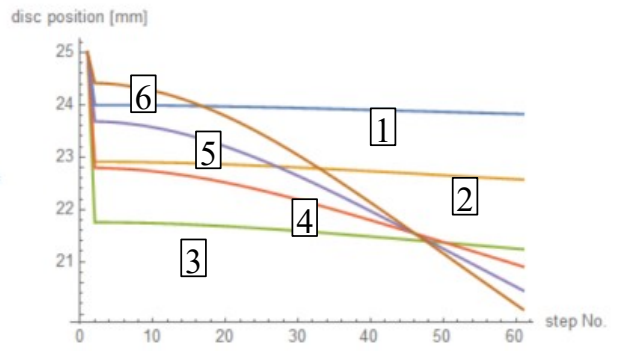

Disc position

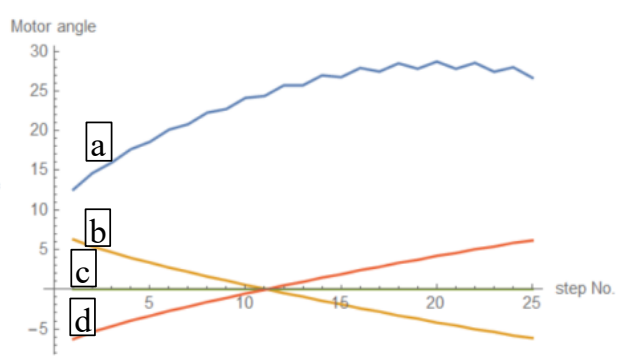

Motor angles (motor-a, b, c, d)

Fig. 23 The end-effector moves straight upward along X-axis $50 \mathrm{~mm}$. 


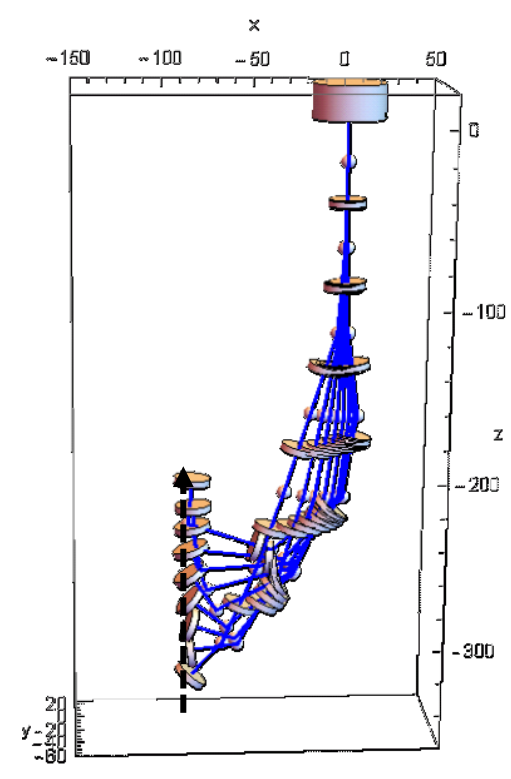

Main view

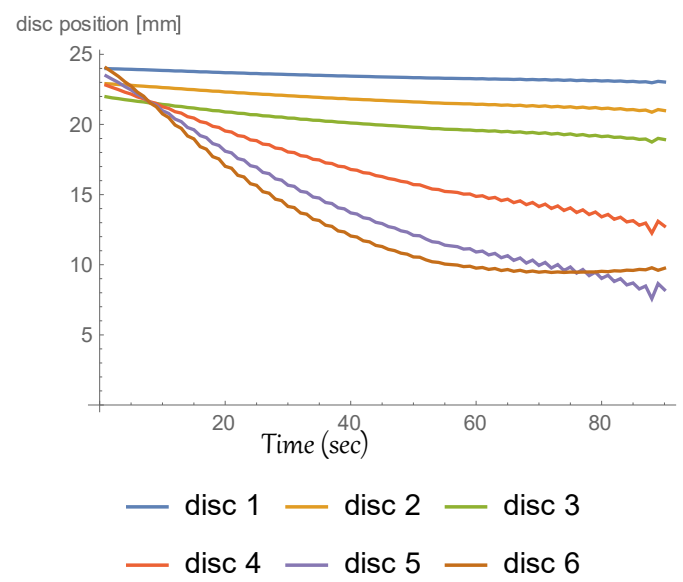

\section{Disc position}

\begin{abstract}
Fig. 24 The end-point moves straight upward along Z-axis $130 \mathrm{~mm}$.
\end{abstract}
According to the obtained results, the sliding backbone disc mechanism engages several segments when bending simultaneously, while the non-sliding backbone bends the actuating segment greatly bend. In order to reach the same bending shape as the sliding one, it requires more torque and power (Fig. 25). To catch the disc displacement by the camera or tracker is very difficult process. Therefore, by conduction of simulation of discs displacement makes possible to prove the concept, for instance in figure 23 where shown disc position displacement and motor angles. 


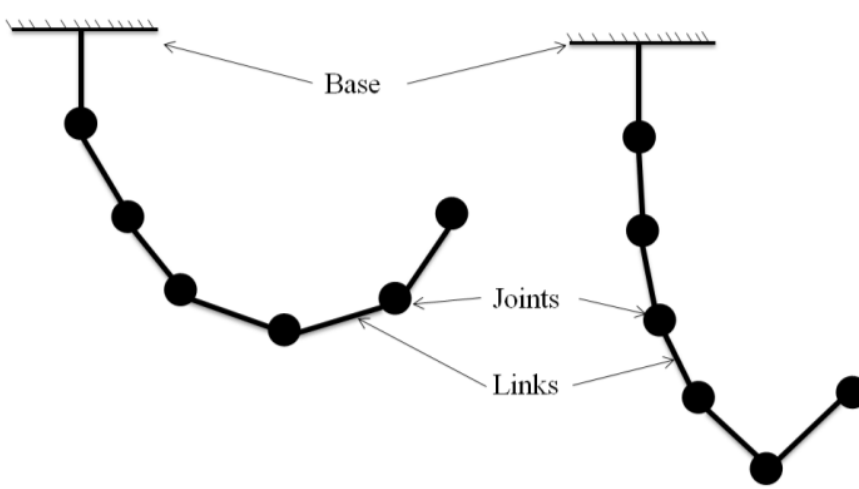

a

b

\section{Test on robot accuracy}

Fig. 25 (a) sliding backbone disk (b) non-sliding backbone disk

To conduct the test on robot accuracy, we designed a task that manipulator should move from point A to point B with a linear trajectory. In this experiment, we utilized a slide potentiometers in the pretension mechanism part, which provides a wire-tension feedback information. By using of such method, we actuated only two of four motors to move the end effector of the manipulator from point A to B (Fig.26). The main task of the experiment, the robot end-effector shold move linearly with less disturbace and fluctuation. To track the end-effector trajectory, we utilesed a camera with tracker. The camera tracked end-effector during the whole experiment.

In figure 27, the graph shows that the sliding prototype demostrates more stable and accurate motion trajectory rather than non-sliding prototype. The main reason of such phenomena is a wire friction along the non-sliding proottype backbone. As in Fig 25, non-sliding prototype concentrates torgue in only single segment and do not distributes the internal bending stress equally.
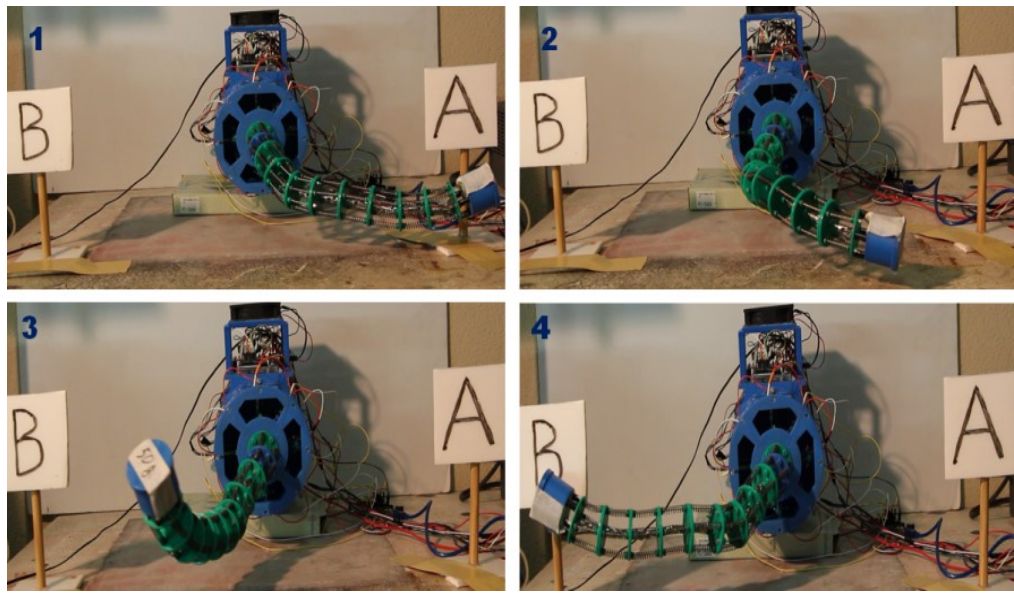

Fig.26 Experimental setup of the accuracy test

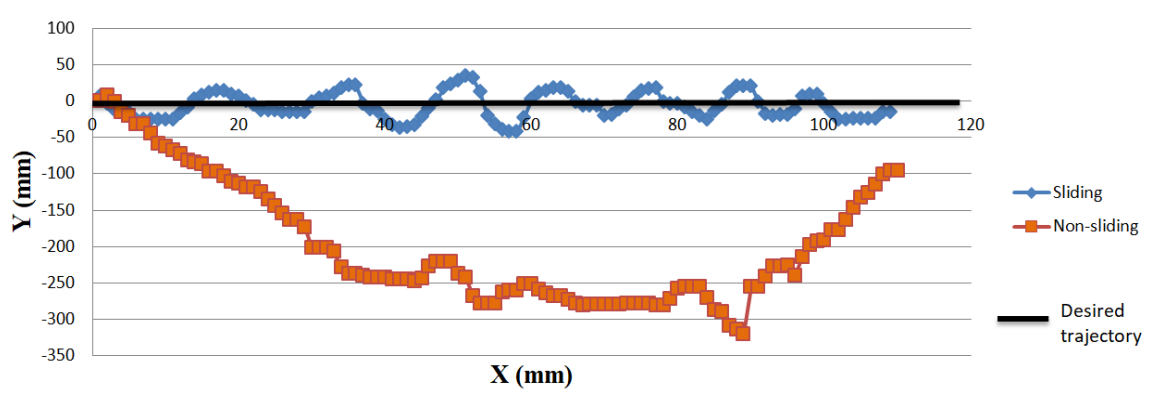

Fig. 27 The graph of accuracy obtained from the tracker

\section{Conclusion and discussion}


This paper presented a wire-driven continuum robot arm design with a passive sliding backbone using a pre-tension mechanism. The kinematics and kinetics validation of the robot were explained and illustrated using simulations. Compared to previous prototypes, we improved robot rigidity and tension control. Based on previous results, we gained necessary information on robot part fabrication and design and continuum robot control methods. Moreover, the proposed pre-tension mechanism device improved robot tension control and helped solve the problem related to sag effect and prevent twist deformation.

According to the obtained findings, the sliding backbone manipulator can reach a sharper bending angle than the previous prototype. The bending discs slide along the slender part by engaging several discs to move simultaneously and distribute the bending stress equally, which makes it possible to achieve such a motion. Furthermore, during the motion, the sliding discs can compensate cable tension in a passive way as well. The proposed design could be one of the best design solutions for continuum manipulators.

In future, we are planning to use a gyro sensor in the tip of the robot to obtain robot orientation and this method will help to simplify kinematics as well. Moreover, the pretension mechanism would be improved as well. From passive mechanism it would be improved as a active mechanism by embedding a linear potentiometers to measure a wire-tension feedback to improve trajectory smoothness.

\section{Supplementary material}

There is a supplementary video material of experiment. The video demonstrates proposed robot general manipulation and payload comparison test. https://www.youtube.com/watch?v=Jvy8z $\mathbf{u} \mathbf{0 B f E \& t = 1 s}$

\section{References}

[1] G. Petroni, M. Niccolini, A. Menciassi, P. Dario, and A. Cuschieri, " A novel intracorporeal assembling robotic system for single-port laparoscopic surgery”, Surgical Endoscopy, vol.27, no. 2,pp. 665-670, February 2013.

[2] J. Wang, S. Wang, J. Li, X. Ren, and R. M. Briggs, “ Development of a novel robotic platform with controllable stiffness manipulation arms for laparoendoscopic single site surgery (LESS)", The International Journal of Medical Robotics and Computer Assisted Surgery, vol. 14, no 1, e1838, February 2018.

[3] R. J. Webster III and B. A. Jones, "Design and kinematic modeling of constant curvature continuum robots: A review", The International Journal of Robotics Research, vol.29, no 13, pp. 1661-1683, June 2010.

[4] Y. Bailly, y. Amirat and G. Fried, "Modelling and Control of a Continuum Style Microrobot for Endovascular Surgery", IEEE Transactions on Robotics, vol 27, no 5, pp. 1024-1030, 2011.

[5] K. Ikuta, Y. Matsuda, D Yajima, and Y. Ota, " Pressure Pulse Drive: A Control Method for the Precise Bending of Hydraulic Active Catheters”, IEEE/ASME Transactions on Mechatronics, vol. 17, no. 5, pp. 876-883, 2012.

[6] P. Dario, M. Carroza, M. Marcacci, S. D’Attanasio. B. Magnami, O. Tonet, and G. Megali, “ A novel mechatronic tool for computer-assisted arthroscopy", IEEE Transactions on Information Technology in Biomedicine, vol. 4, no. 1, pp. 15-29, 2000.

[7] H. Choset, M. Zenati, T. Ota, A. Degani, D. Schwartzman, B. Zubiate, and C. Wright, “ Enabling Medical Robotics for the Next Generation of Minimally Invasive Procedures: Minimally Invasive Cardiac Surgery with Single Port Access", in Surgical Robotics -Systems Applications and Visions, J Rosen. B. Hannaford, and R. M. Satava, Eds., 2011, pp 257-270.

[8] I. Gravagne, C, Rahn, and I Walker, "Large deflection dynamics and control for planar continuum robots", IEEE/ASME Transactions on Mechatronics, vol. 8 no. 2, pp. 299-307, 2003.

[9] N. Simaan, K. Xu, A. Kapoor, W. Wei, P. Kazanzides, P. Flint, and R. Taylor. “ Design and Integration of a Telerobotic System for Minimally Invasive Surgery of the Throat", The International Journal of Robotics Research, vol. 28, no. 9, pp. 1134-1153, 2009.

[10]A. Yeshmukhametov, Z Buribayev, Y. Amirgaliyev and R. Ramakrishnan, "Modelling and Validation of New Continuum Robot Backbone Design with Variable Stiffness Inspired from elephant Trunk", International Conference on Mechanics and Mechatronics Research, IOP conference series: Material Science and Engineering 417 (2018)012010. 
[11] A. Yeshmukhametov, Z. Buribayev, Y. Amirgaliyev and B. Amirgaliyev, "A Bio-inspired a novel continuum robot arm with variable backbone design: Modelling and Validation", Journal of Theoretical and Applied Information Technology, vol. 97, issue 18. October 2019.

[12] J. Starke, E. Amanov, M. Taha, J. Burgner -Kahrs, "On the Merits of Helical Tendon Routing in Continuum Robots", IEEE/RSJ International Conference on Intelligent Robots and Systems (IROS), 2017, Vancouver, Canada.

[13]A. Yeshmukhametov, K. Koganezawa and Y. Yamamoto. "A Novel Discrete Wire-Driven Continuum Robot Arm with Passive Sliding Disc: Design, Kinematics and Passive Tension Control”, Robotics, MDPI, July, 2019; doi:10.3390/robotics8030051.

[14] A. Yeshmukhametov, K. Koganezawa and Y. Yamamoto, "Design and Kinematics of CableDriven Continuum Robot Arm with Universal Joint Backbone", IEEE International Conference on Robotics and Biomimetics, December, Kuala-Lumpur, 2018

[15]I.D. Walker and M.W. Hanna, “ A novel 'elephant's trunk' robot”, IEEE/ASME International Conference on Advanced and Intelligent Mechatronics 1999, DOI:10.1109/AIM.1999.803204

[16]N. Thien-Dang and J. Burgner-Kahrs, "A Tendon-Driven Continuum Robot with Extensible Sections", IEEE/RSJ International Conference on Intelligent Robots and Systems (IROS), Hamburg, Germany, 2015.

[17] Kang, B.; Koijev, R.; Sinibaldi, E. The First Interlaced Continuum Robot, Devised to Intrinsically Follow the Leader. PLoS One 2016, 11, e0150278, Doi:10.1371journal.pone.0150278.

[18] Ji, D.; Kang, T.H.; Shim, S.; Lee, S.; Hong, J. Wire-driven flexible manipulator with constrained spherical joints for minimally invasive surgery. In International Journal of Computer Assisted Radiology and Surgery. Springer: Berlin, Germany, 2019; pp. 1-13.

[19]Zhao, B.; Zhang, W.; Zhang, Z.; Zhu, X.; Xu, K. Continuum Manipulator with Redundant Backbones and Constrained Bending Curvature for Continuously Variable Stiffness. In Proceedings of the IEEE International Conference on Intelligent Robots and Systems (IROS), Madrid, Spain, 1-5 October 2018; pp. 7492-7499.

[20] M.W. Hannan and I.D. Walker. "Kinematics and the Implementation of an Elephant's Trunk Manipulator and other Continuum Style Robots". In Journal of Robotic Systems. 20(2). Pp. 45-63, 2003.

[21] Webster, R.J.; Jones, B.A. Design and kinematic modelling of constant curvature continuum robots: A review. Int. J. Robot. Res. 2010, 29, 1661-1683.

[22] I.D. Walker and M. W. Hannan. “A Novel 'Elephant's Trunk' robot,” In Proceedings IEEE/ASME International Conference on Advanced Intelligent Mechatronics, Atlanta, GA, USA. Pp. 410-415, 1999.

[23] H.Ohno and S. Hirose. "Design of Slim Slime Robot and its Gait of Locomotion", In proceedings of 2001 IEEE/RSJ International Conference on Intelligent Systems, Maui, Hawaii, USA. Pp. 707-715, 2001.

[24]I.A. Gravagne. “ Design, Analysis and Experimentation: The Fundamentals of Continuum Robotic Manipulators”, Ph.D. Dissertation, Department of Electrical and Computer Engineering, Clemson University, August 2002.

[25]Zheng Li et.al., "A novel constrained wire-driven flexible mechanism and its kinematic analysis", Mechanism and Machine Theory 95 (2016)59-75. Doi: 10.1016/j.mechmachtheory.2015.08.019.

[26] A. Yeshmukhametov, K. Koganezawa, A. Seidakhmet and Y. Yamamoto, "A Novel Passive Pretension Mechanism for Wire-Driven Continuum Manipulators", In proceedings of IEEE International Symposium On System Integration (SII2020), Honolulu, Hawaii, USA, January 2020. (accepted paper)

[27] S.M. Hadi Sadati, S. Elnaz Naghibi, Ali Shiva, I.D. Walker, K. Althoefer, and T. Nanayakkara, “ Mechanics of Continuum Manipulators, A comparative Study Methods with Experiments" Lecture Notes in Computer Science, July 2017 